\title{
Alopecuroides A-E, Matrine-Type Alkaloid Dimers from the Aerial Parts of Sophora
}

alopecuroides

Chun-Lin Fan, ${ }^{\dagger, \S}$ Yu-Bo Zhang, ${ }^{\dagger, \S}$ Ye Chen, ${ }^{\dagger, \S}$ Pei Xie,,${ }^{\ddagger}$ Guo-Cai Wang,,${ }^{\dagger}$ Hai-Yan Tian, ${ }^{\dagger}$ Yao-Lan Li, ${ }^{\dagger}$ Xiao-Jun Huang, ${ }^{\dagger}$ Xiao-Qi Zhang, ${ }^{\dagger}$ Zhi-Yong Li, ${ }^{\#}$ Jun-Shan Liu, ${ }^{*}$, Wen-Cai Ye, ${ }^{*}, \dagger$ and Wei-Min Chen $*, \dagger$

'College of Pharmacy, Jinan University, Guangzhou 510632, People's Republic of China

\#School of Traditional Chinese Medicine, Southern Medical University, Guangzhou 510515, People’s

Republic of China

${ }^{\#}$ School of Pharmacy, Yantai University, Yantai 264005, People's Republic of China 
General Apparatus and Chemicals .................................................................................................................1

Extraction and Isolation .....................................................................................................................................

X-ray Diffraction Data Analysis ..............................................................................................................2

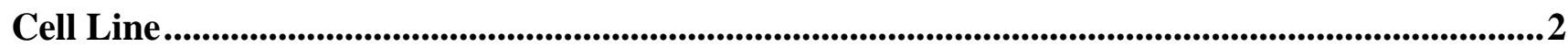

3-(4,5-dimethylthiazol-2-yl)-2,5-diphenyltetrazolium bromide (MTT) Assay ...................................3

Enzyme-linked Immunosorbent Assay (ELISA) ..........................................................................................3

Statistical Analysis ..............................................................................................................................................

Figure S1. HRESIMS spectrum of compound 1 ....................................................................................

Figure S2. UV spectrum of compound 1 ...................................................................................................4

Figure S3. IR spectrum of compound 1 .......................................................................................................5

Figure S4. ${ }^{1} \mathrm{H}$ NMR spectrum of compound 1.................................................................................................5

Figure S5. ${ }^{13} \mathrm{C}$ NMR spectrum of compound 1.........................................................................................6

Figure S6. DEPT-135 spectrum of compound 1 .................................................................................6

Figure S7. ${ }^{1} \mathrm{H}-{ }^{1} \mathrm{H}$ COSY spectrum of compound 1 ................................................................................

Figure S8. HSQC NMR spectrum of compound 1......................................................................................

Figure S9. HMBC NMR spectrum of compound 1..................................................................................8

Figure S10. NOESY spectrum of compound 1 ...............................................................................................8

Figure S11. HRESIMS spectrum of compound 2 ...................................................................................9

Figure S12. UV spectrum of compound 2 .....................................................................................................9

Figure S13. IR spectrum of compound 2 ......................................................................................................10

Figure S14. ${ }^{1} \mathrm{H}$ NMR spectrum of compound 2........................................................................................10

Figure S15. ${ }^{13} \mathrm{C}$ NMR spectrum of compound 2....................................................................................11

Figure S16. DEPT-135 spectrum of compound 2 ...................................................................................11

Figure S17. ${ }^{1} \mathrm{H}-{ }^{1} \mathrm{H}$ COSY spectrum of compound 2................................................................................12

Figure S18. HSQC NMR spectrum of compound 2.................................................................................12

Figure S19. HMBC NMR spectrum of compound 2................................................................................13

Figure S20. NOESY spectrum of compound 2 .....................................................................................13 
Figure S21. HRESIMS spectrum of compound 3 .

Figure S22. UV spectrum of compound 3 .......................................................................................14

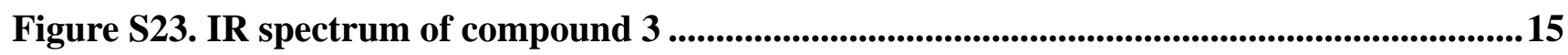

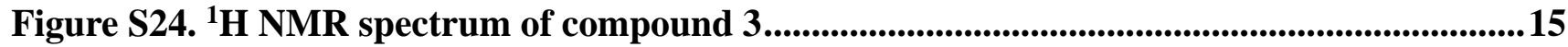

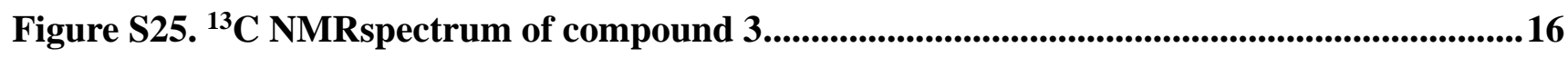

Figure S26. DEPT-135 spectrum of compound 3 .............................................................................

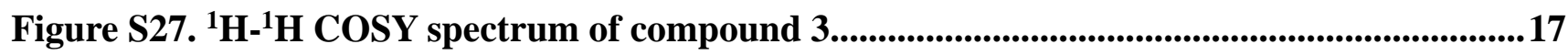

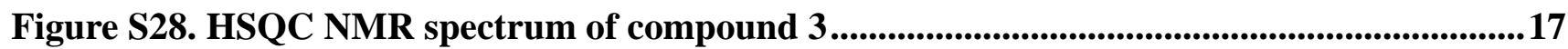

Figure S29. HMBC NMR spectrum of compound 3..............................................................................18

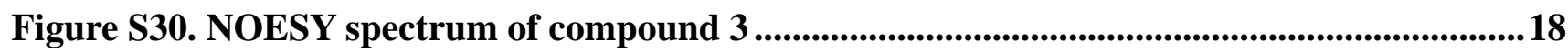

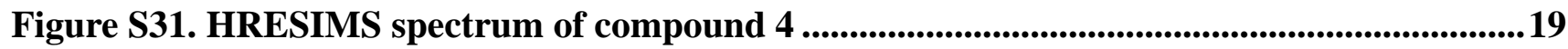

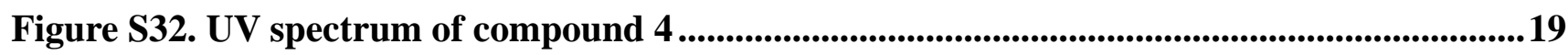

Figure S33. IR spectrum of compound $4 \ldots \ldots \ldots$

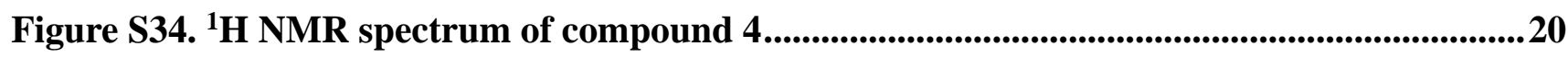

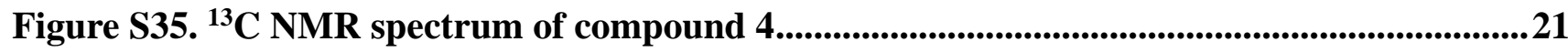

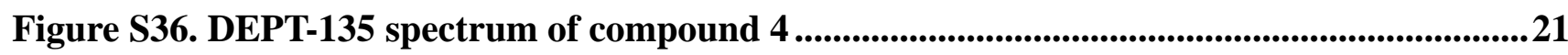

Figure S37. ${ }^{1} \mathrm{H}-{ }^{1} \mathrm{H}$ COSY spectrum of compound 4 .............................................................................22

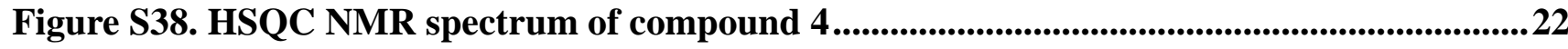

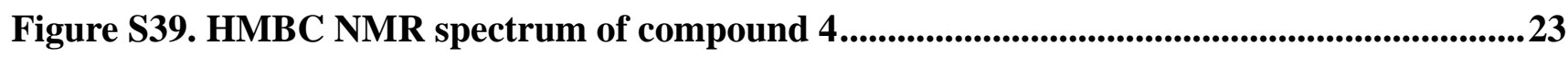

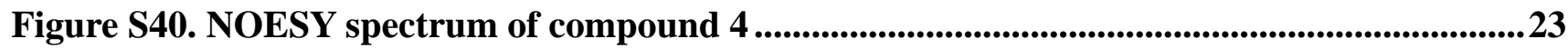

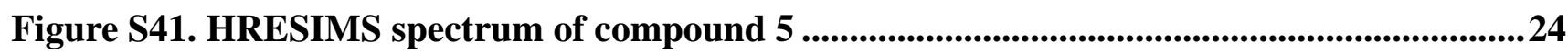

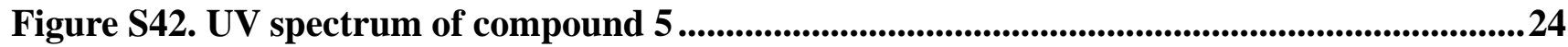

Figure S43. IR spectrum of compound 5 .......................................................................................................224

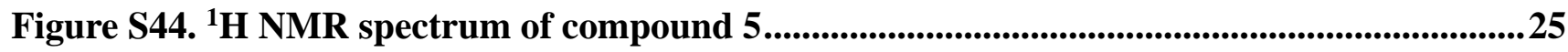

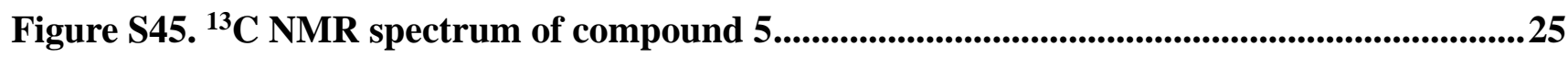

Figure S46. DEPT-135 spectrum of compound 5 ................................................................................26

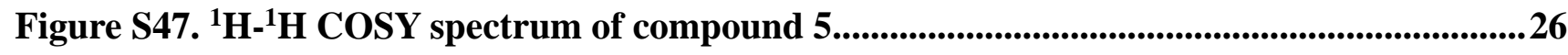

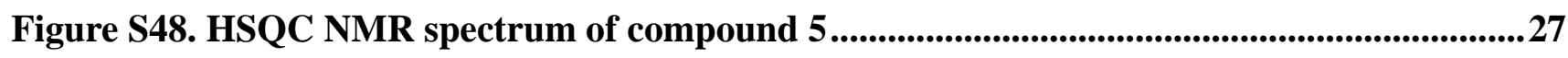

Figure S49. HMBC NMR spectrum of compound 5

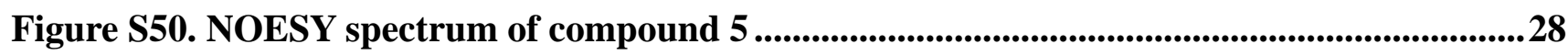


General Apparatus and Chemicals. Melting points were measured on a BÜCHI Melting Point B-545 (BÜCHI, Flawil, Switzerland). Optical rotations were recorded on a JASCO P-1020 polarimeter (JASCO, Tokyo, Japan). UV spectra were recorded on a JASCO V-550 UV/Vis spectrophotometer (JASCO, Tokyo, Japan). IR spectra were determined on a JASCO FT/IR-480 plus Fourier transform infrared spectrometer (JASCO, Tokyo, Japan) using KBr pellets. HRESIMS data were recorded on an Agilent 6210 MSD TOF mass spectrometer (Agilent technologies, CA, USA). NMR spectra were recorded on AV-500 spectrometers (Bruker, fallanden, Switzerland). Analytical HPLC was performed on Agilent 1260 Infinity with a DAD detector (Agilent technologies, CA, USA) and an analytical column (Waters XBridge, $5 \mu \mathrm{m}, 4.6 \times 250 \mathrm{~mm}$ ). Preparative HPLC was performed on Agilent 1260 Infinity equipped with an UV detector (Agilent technologies, CA, USA) and a semi-preparative column (Waters XBridge, $5 \mu \mathrm{m}, 10 \times 250 \mathrm{~mm}$ ). Thin-layer chromatography (TLC) was performed using precoated silica gel plates (GF254, Qingdao, P. R. China). Open column chromatography (CC) was performed using macroporous resin (AB-8, Tianjin, P. R. China), MCI gel (CHP20/P120, Mitsubishi, Japan), ODS (Merck, Darmstadt, Germany) and Sephadex LH-20 (Pharmacia Biotec AB, Sweden). IL-6 and TNF- $\alpha$ ELISA kits were purchased from Dakewei (Beijing, China). Lipopolysaccharides (LPS) were purchased from Sigma-Aldrich (St Louis, MO). All reagents used were analytical or chromatographic grade.

Extraction and isolation. The dried aerial parts of $S$. alopecuroides $(50.0 \mathrm{~kg})$ were powdered and extracted three times with $95 \% \mathrm{EtOH}$ at room temperature. The extract was suspended in $\mathrm{H}_{2} \mathrm{O}$ and then acidified with $1 \% \mathrm{HCl}$ to $\mathrm{pH} 3$. The neutral components were removed by extraction with $\mathrm{CH}_{2} \mathrm{Cl}_{2}$. The remaining aqueous layer was adjusted to $\mathrm{pH} 9$ with $\mathrm{NH}_{3} \cdot \mathrm{H}_{2} \mathrm{O}$ and re-extracted with $\mathrm{CH}_{2} \mathrm{Cl}_{2}$ to afford a total alkaloids (724.6 g). Total alkaloids was subjected to column chromatography 
(CC) over macroporous resin (AB-8) eluted with a gradient system of $\mathrm{EtOH} / \mathrm{H}_{2} \mathrm{O}$ (from 0:1 to 95:5) to give four fractions (A to D). Fraction D was separated by a MCI gel CC $\left(\mathrm{MeOH}-\mathrm{H}_{2} \mathrm{O}, 20: 80\right.$ to 100:0) to afford 11 fractions (D-1 to D-11). Fraction D-5 was separated by Sephadex LH-20 CC $(\mathrm{MeOH})$ to yield 6 fractions (D-5-1 to D-5-6). Fraction D-5-3 was separated by an ODS CC using a $\mathrm{MeOH}-\mathrm{H}_{2} \mathrm{O}$ mixture as the eluent to yield 8 fractions (D-5-3-1 to D-5-3-8). Fraction D-5-3-2 was further purified by semi-preparative HPLC applying $60 \% \mathrm{MeOH}$ (with $0.02 \% \mathrm{Et}_{2} \mathrm{NH}$ added to both solvents) to yield compounds $\mathbf{1}(14.6 \mathrm{mg})$ and 2 (12.5 mg). Fraction D-5-3-3 was further separated by an ODS CC to afford 5 fractions (D-5-3-3-1 to D-5-3-3-5), and then fraction D-5-3-3-4 was purified by semi-preparative HPLC applying $35 \% \mathrm{CH}_{3} \mathrm{CN}$ (with $0.02 \% \mathrm{Et}_{2} \mathrm{~N}$ added to both solvents) to yield compound 5 (18.6 mg). Fraction D-9 was further separated by an ODS column using a $\mathrm{MeOH}-\mathrm{H}_{2} \mathrm{O}$ mixture as the eluent to yield 3 fractions (D-9-1 to D-9-3). Compound 3 (18.9 mg) was recrystallized from fraction D-9-2. Fraction D-9-3 was purified by semi-preparative $\mathrm{HPLC}$ applying a 55\% $\mathrm{CH}_{3} \mathrm{CN}$ (with $0.02 \% \mathrm{Et}_{2} \mathrm{NH}$ added to both solvents) to yield compound 4 (20.5 mg).

X-ray Diffraction Data Analysis. X-ray crystallographic data for 1-5 were measured on an Agilent Gemini S Ultra CCD diffractometer using $\mathrm{Cu} \mathrm{K \alpha}$ radiation. The structures were solved by direct methods using SHELXT and refined with full-matrix least-squares calculations on $F^{2}$ using SHELXL via OLEX2. Crystallographic data for 1-5 have been deposited in the Cambridge Crystallographic Data Centre (CCDC, https://www.ccdc.cam.ac.uk/) and the CCDC numbers are 1522211, 1879302, 1879402, 1521002 and 1502007, respectively.

Cell Line. Murine macrophage cell line RAW 264.7 was obtained from American Type Culture Collection (ATCC, Rockville, MD). Cells were cultured in 1640 supplemented with $10 \%$ (v/v) fetal bovine serum (Gibco) and $1 \%(v / v)$ penicillin/streptomycin $(\mathrm{Gibco})$ at $37^{\circ} \mathrm{C}$ in a humidified incubator 
with $5 \% \mathrm{CO}_{2}$.

3-(4,5-dimethylthiazol-2-yl)-2,5-diphenyltetrazolium Bromide (MTT) Assay. RAW 264.7 cells were cultured in a 96-well plate at the density of $5 \times 10^{4}$ cells $/ \mathrm{mL}$ for $24 \mathrm{~h}$. Then, cells were treated with various concentrations of compounds in the presence or absence of LPS $(1 \mu \mathrm{g} / \mathrm{mL})$ for another $24 \mathrm{~h} .30 \mu \mathrm{L}$ of MTT $(5 \mathrm{mg} / \mathrm{mL})$ was added to incubate for $4 \mathrm{~h}$ at $37^{\circ} \mathrm{C}$. The supernatant was discarded and the formazan crystal was dissolved with DMSO (100 $\mu \mathrm{L} /$ well). The absorbance was measured at $570 \mathrm{~nm}$ using a microplate reader (Thermo Scientific).

Enzyme-linked Immunosorbent Assay (ELISA). To quantify the levels of proinflammatory cytokines, the RAW264.7 cells were placed in a 24-well plate at a density of $2 \times 10^{5} / \mathrm{mL}$. After cultured for $24 \mathrm{~h}$, cells were pretreated with $50 \mu \mathrm{M}$ of compounds 1-5 (non-toxic concentration) for 2 $\mathrm{h}$ before stimulation with LPS for another $16 \mathrm{~h}$. The concentrations of IL- 6 and TNF- $\alpha$ in cell supernatants were quantified with ELISA kits according to the manufacturers' instructions.

Statistical Analysis. Data were expressed as mean \pm SD. Difference between groups was determined by Student's t-test (two-tailed). Statistical significance was considered when $P$ values $<0.05$. 


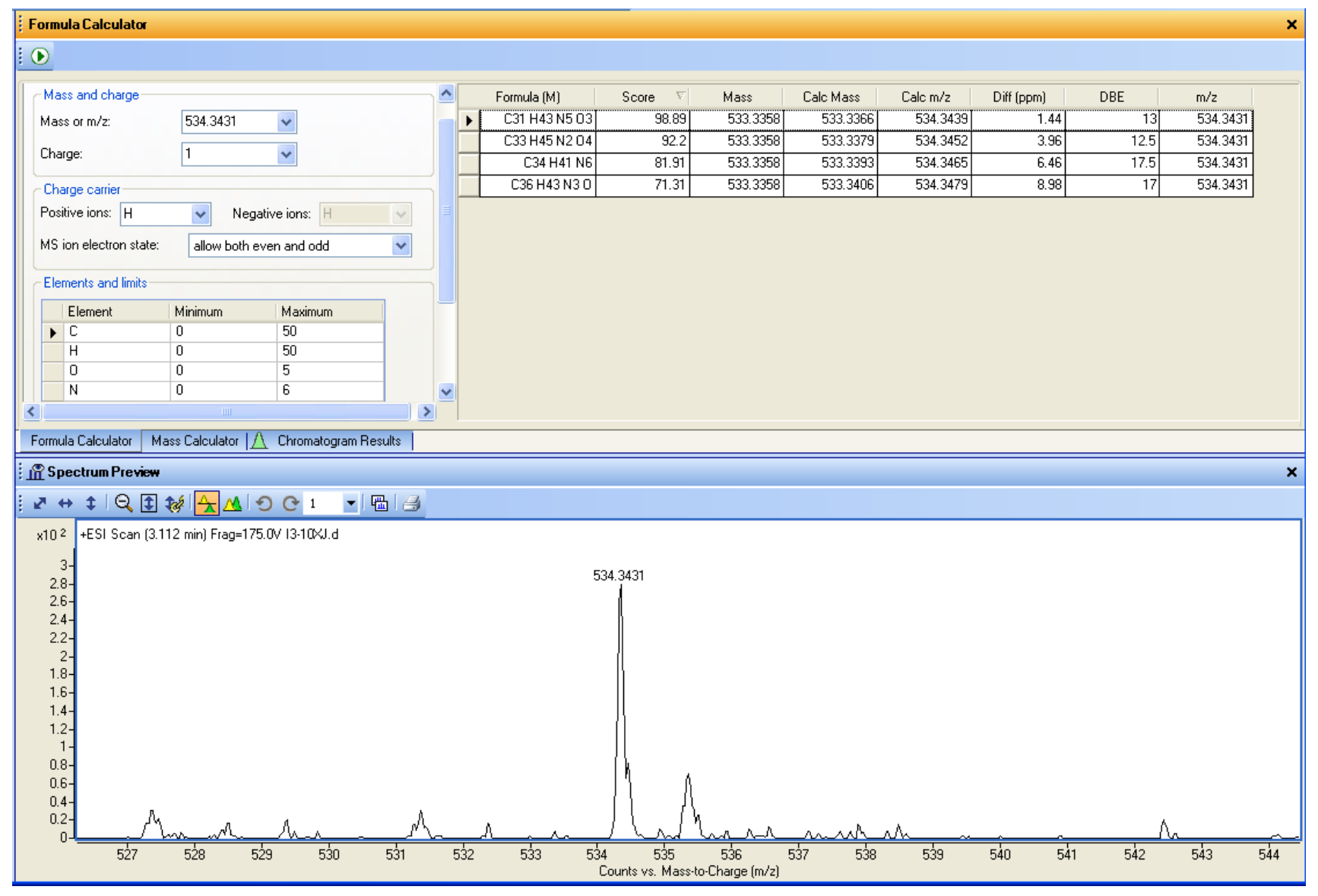

Figure S1. HRESIMS spectrum of compound 1

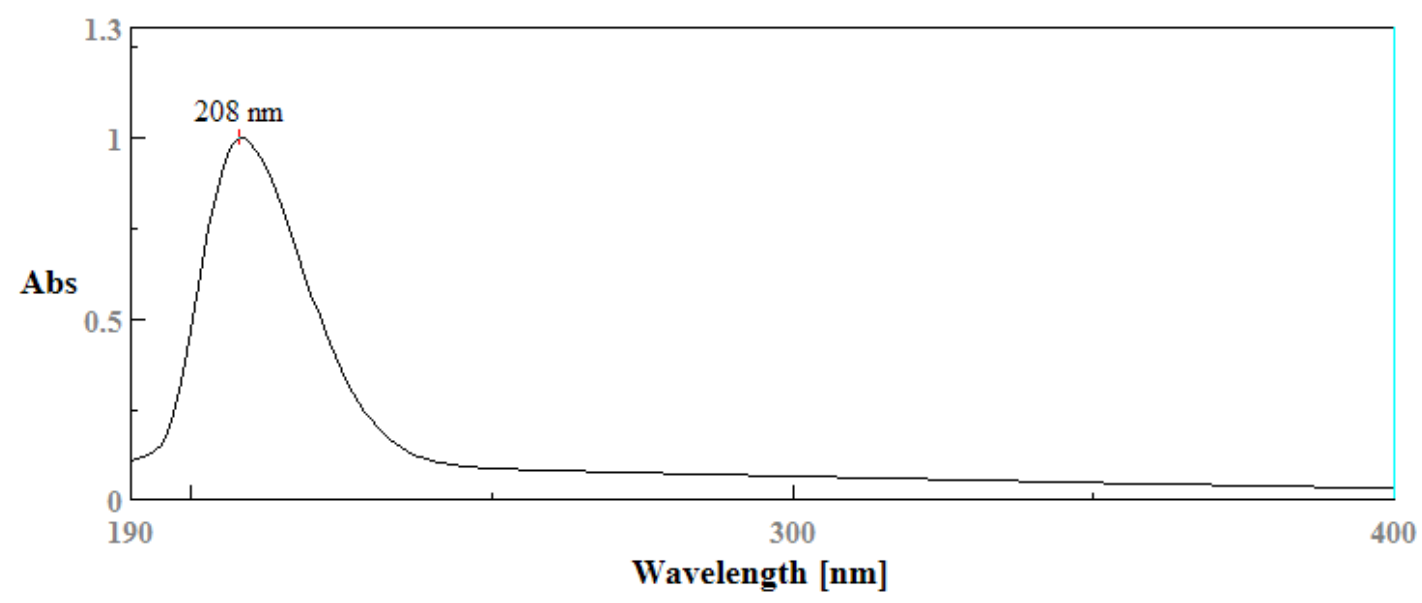

Figure S2. UV spectrum of compound 1 


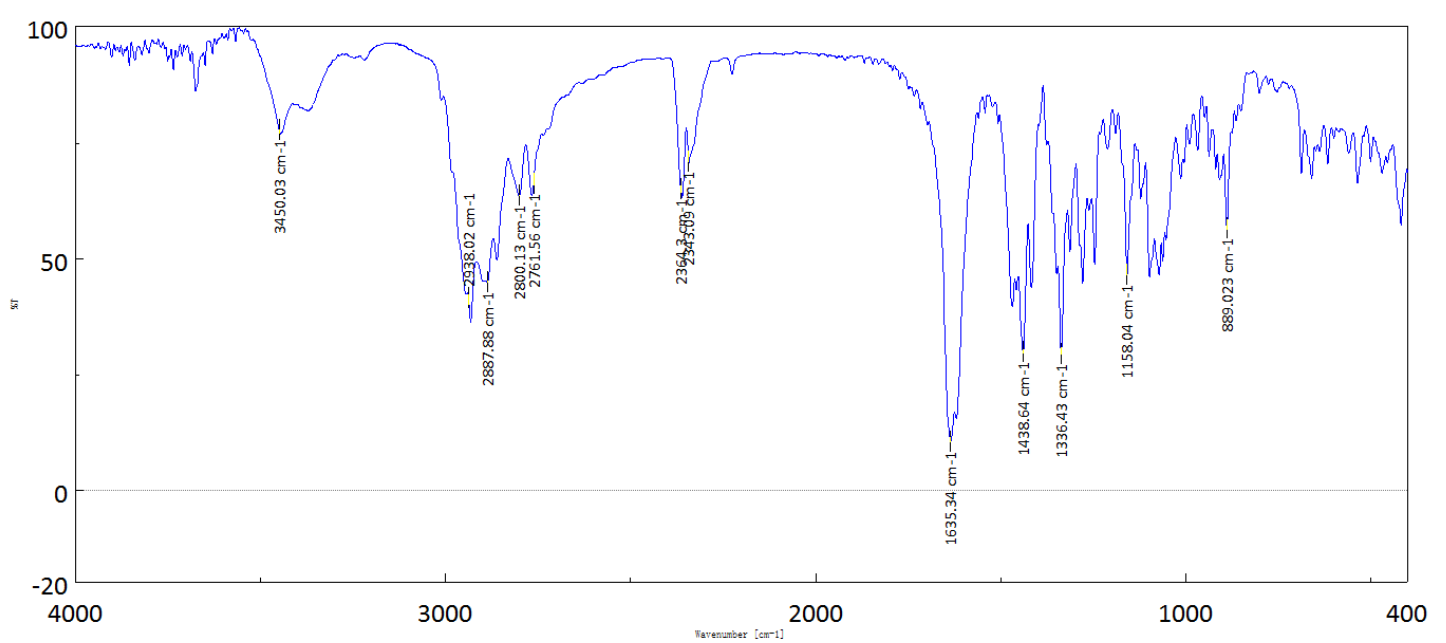

Figure S3. IR spectrum of compound 1

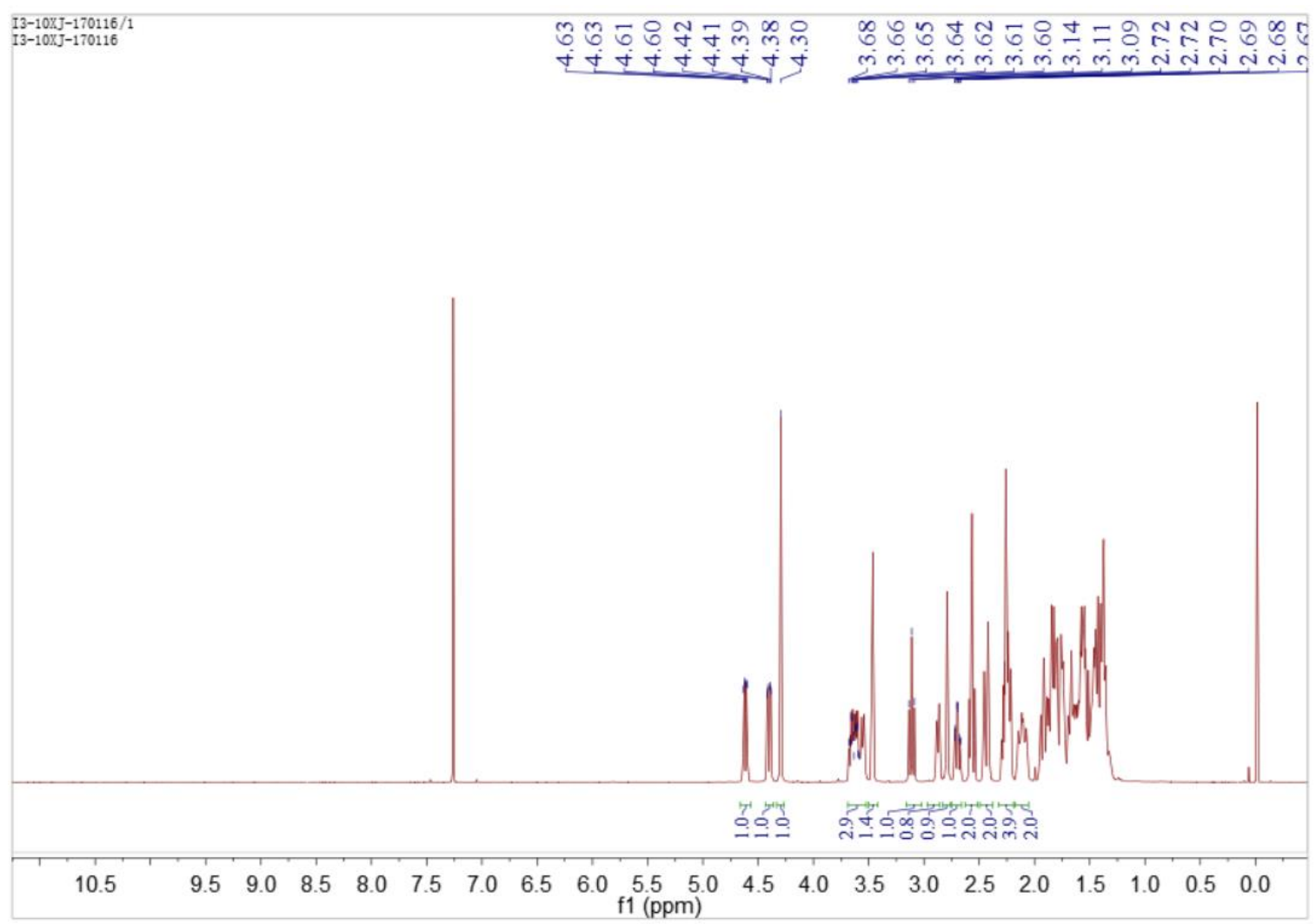

Figure S4. ${ }^{1} \mathrm{H}$ NMR spectrum of compound 1 


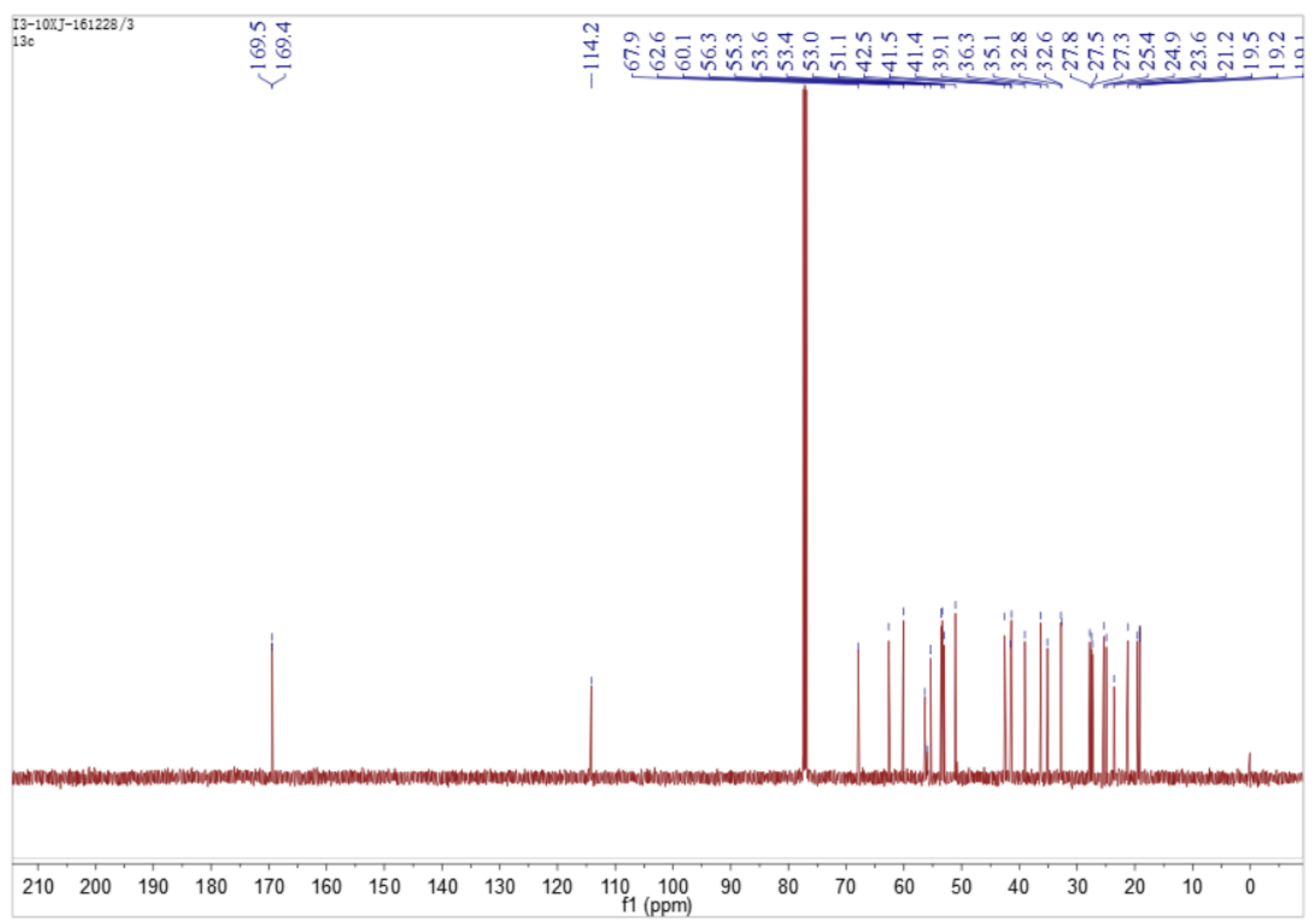

Figure S5. ${ }^{13} \mathrm{C}$ NMR spectrum of compound $\mathbf{1}$

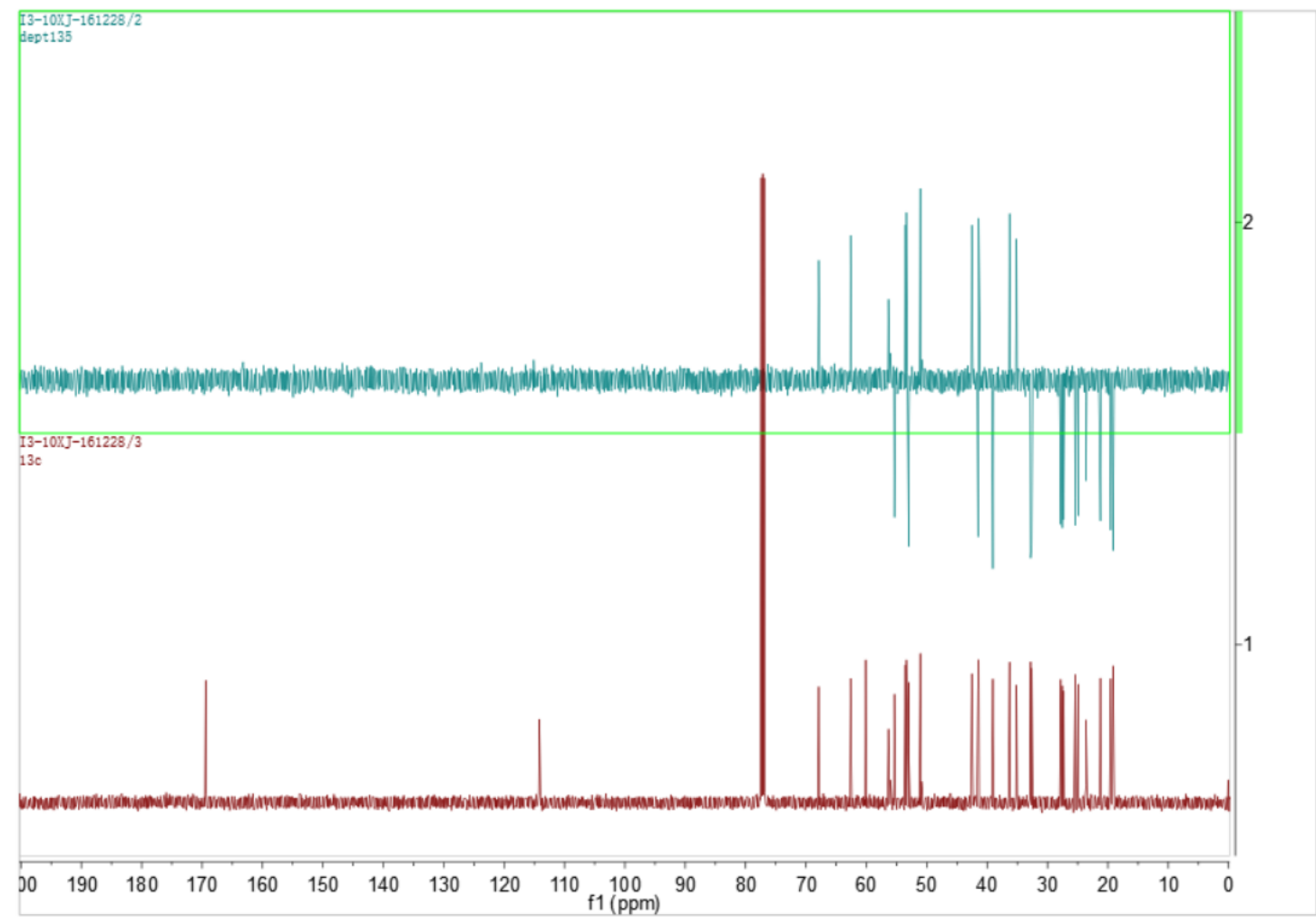

Figure S6. DEPT-135 spectrum of compound 1 


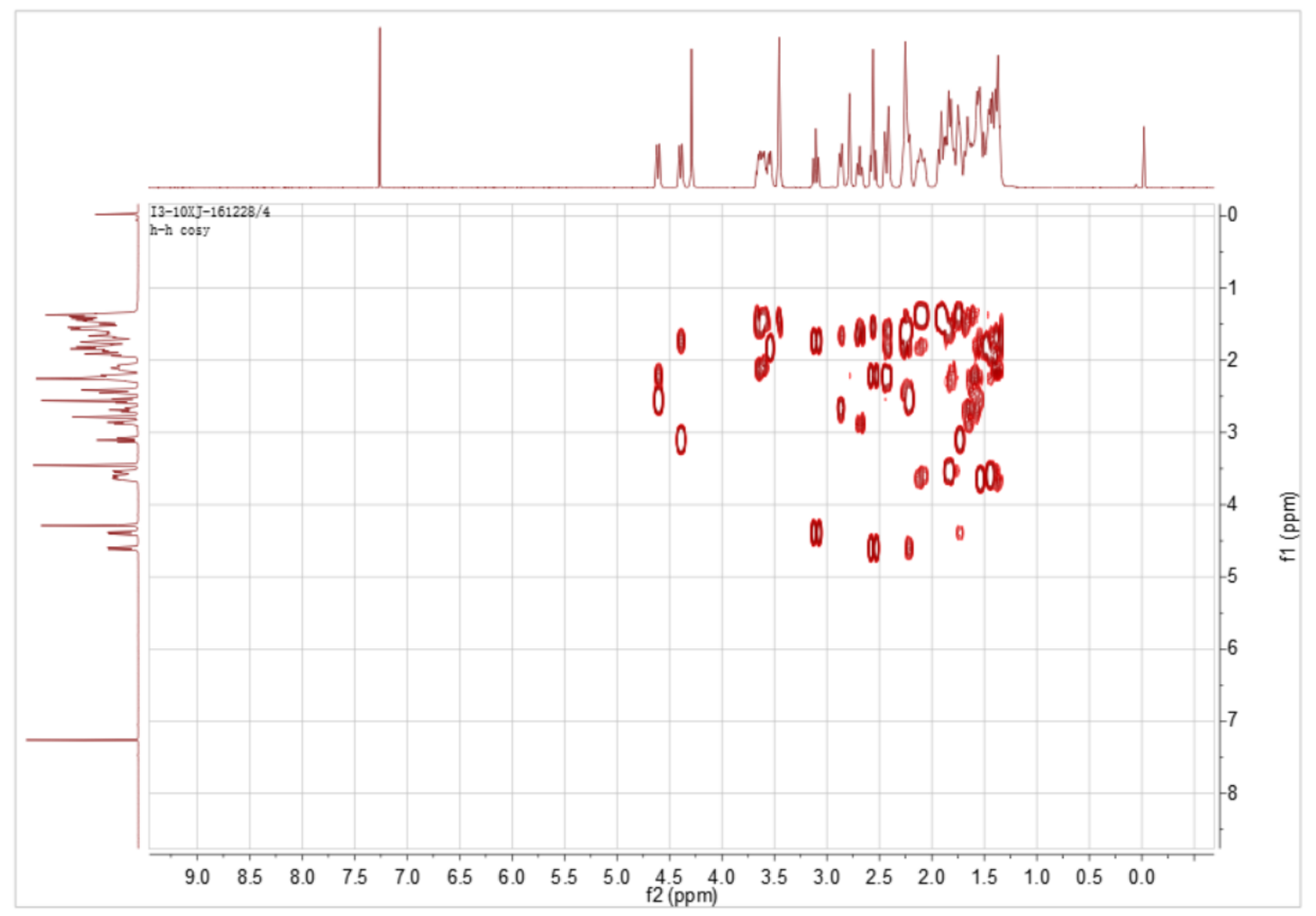

Figure S7. ${ }^{1} \mathrm{H}-{ }^{1} \mathrm{H}$ COSY spectrum of compound 1

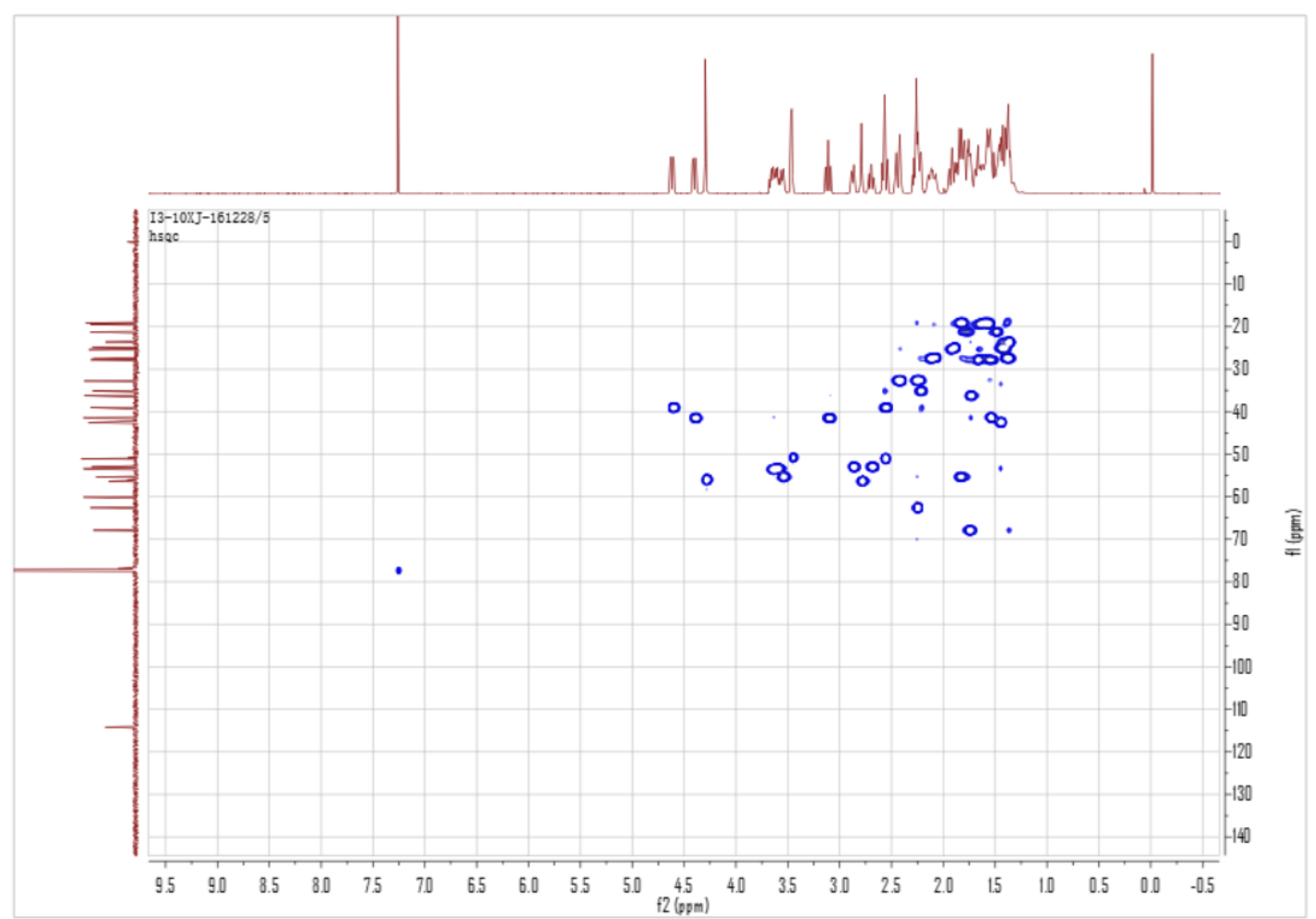

Figure S8. HSQC NMR spectrum of compound 1 


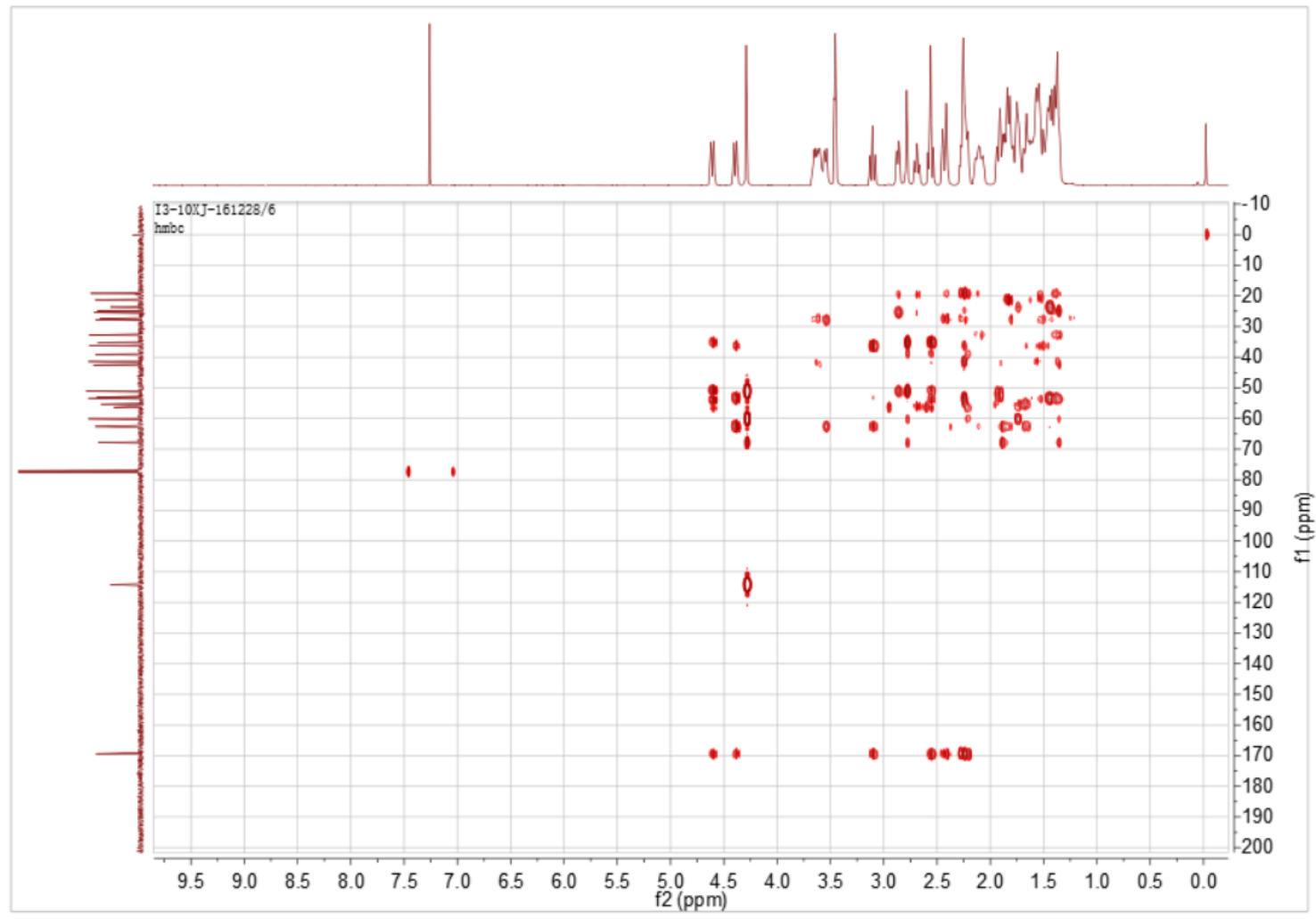

Figure S9. HMBC NMR spectrum of compound 1

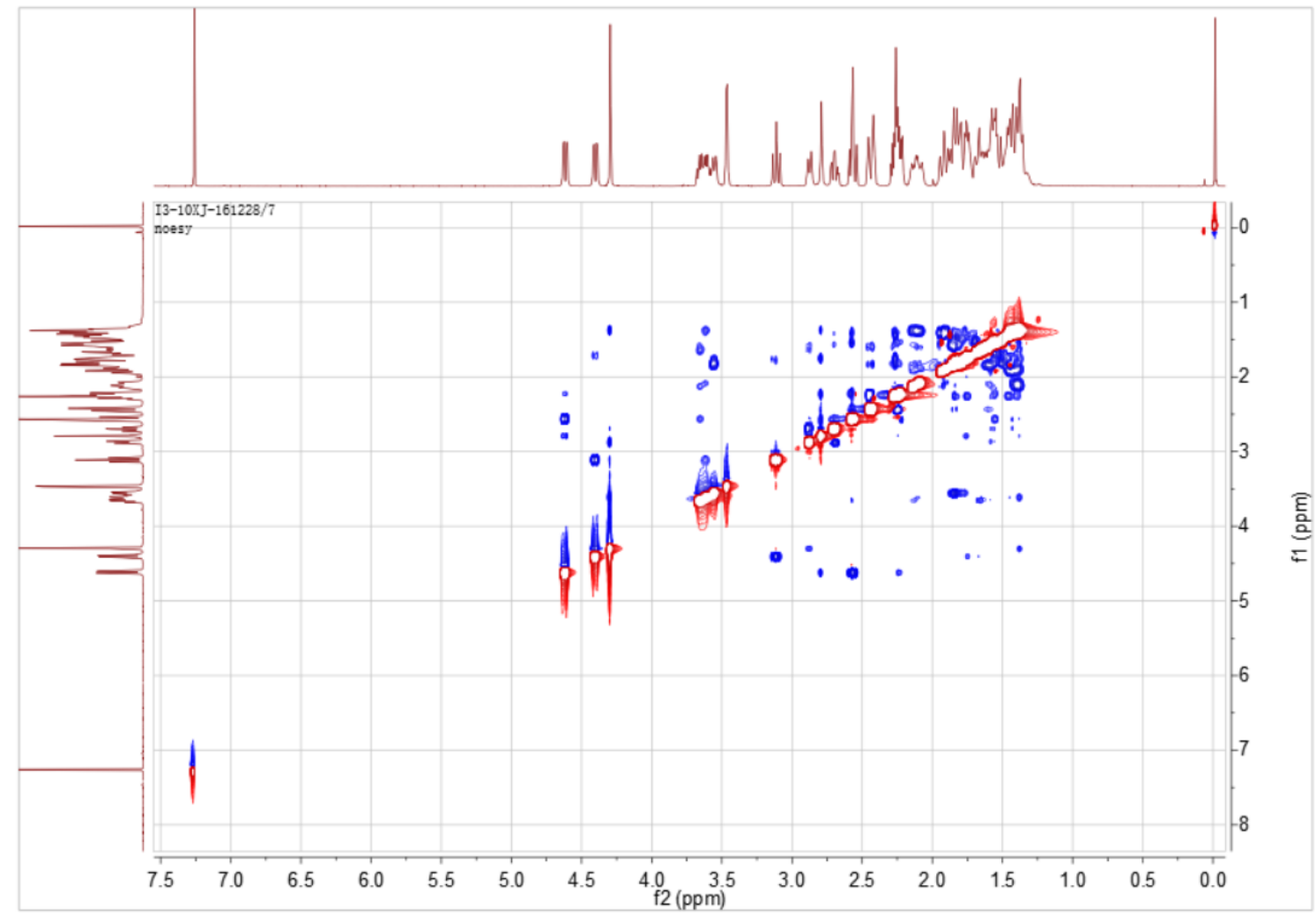

Figure S10. NOESY spectrum of compound 1 


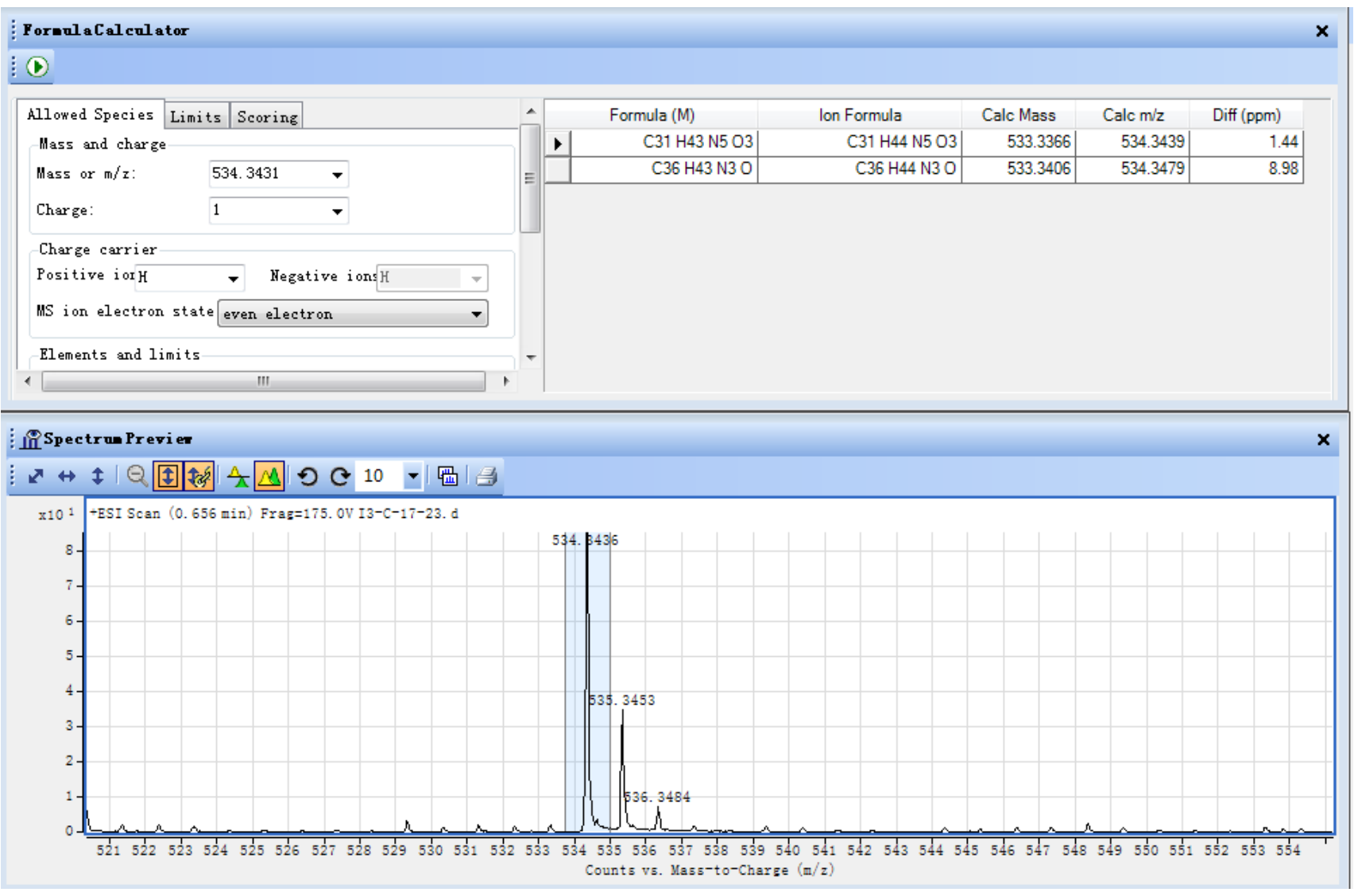

Figure S11. HRESIMS spectrum of compound 2

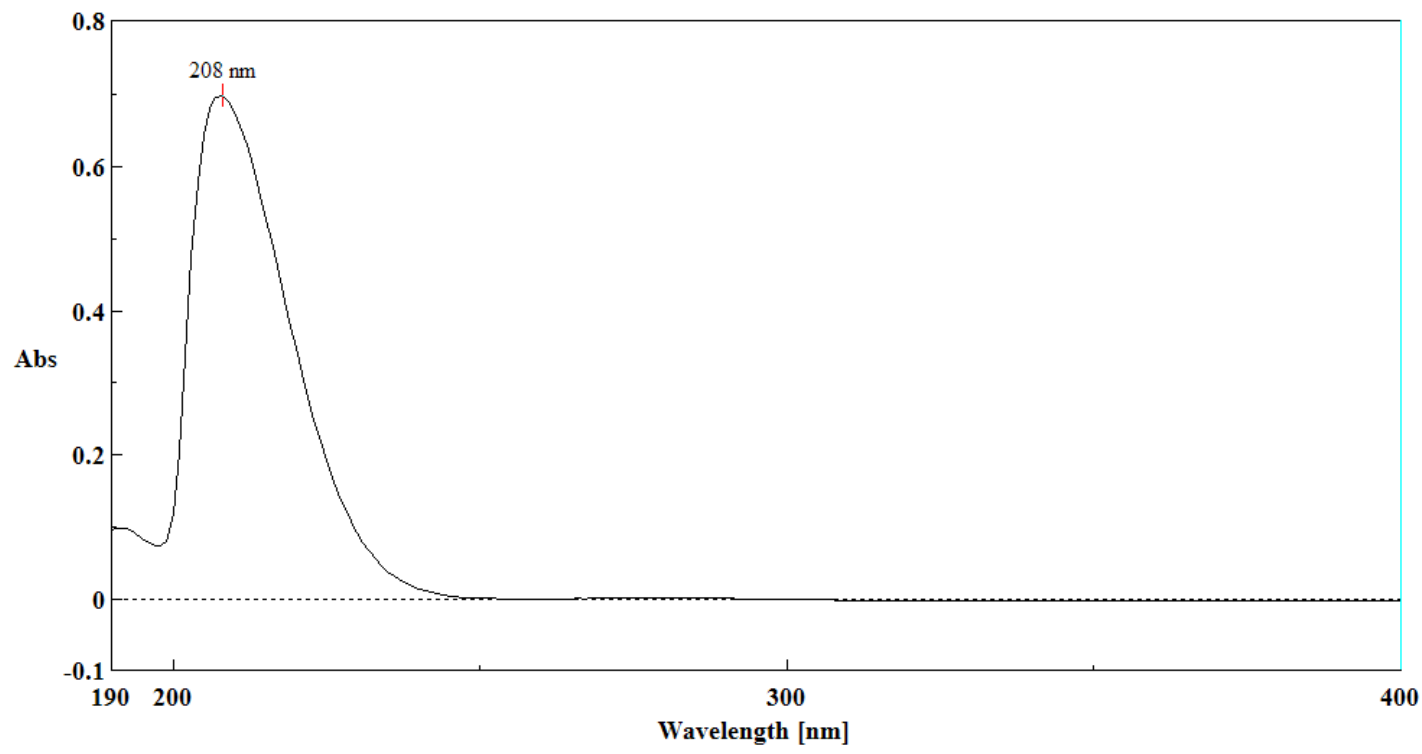

Figure S12. UV spectrum of compound 2 


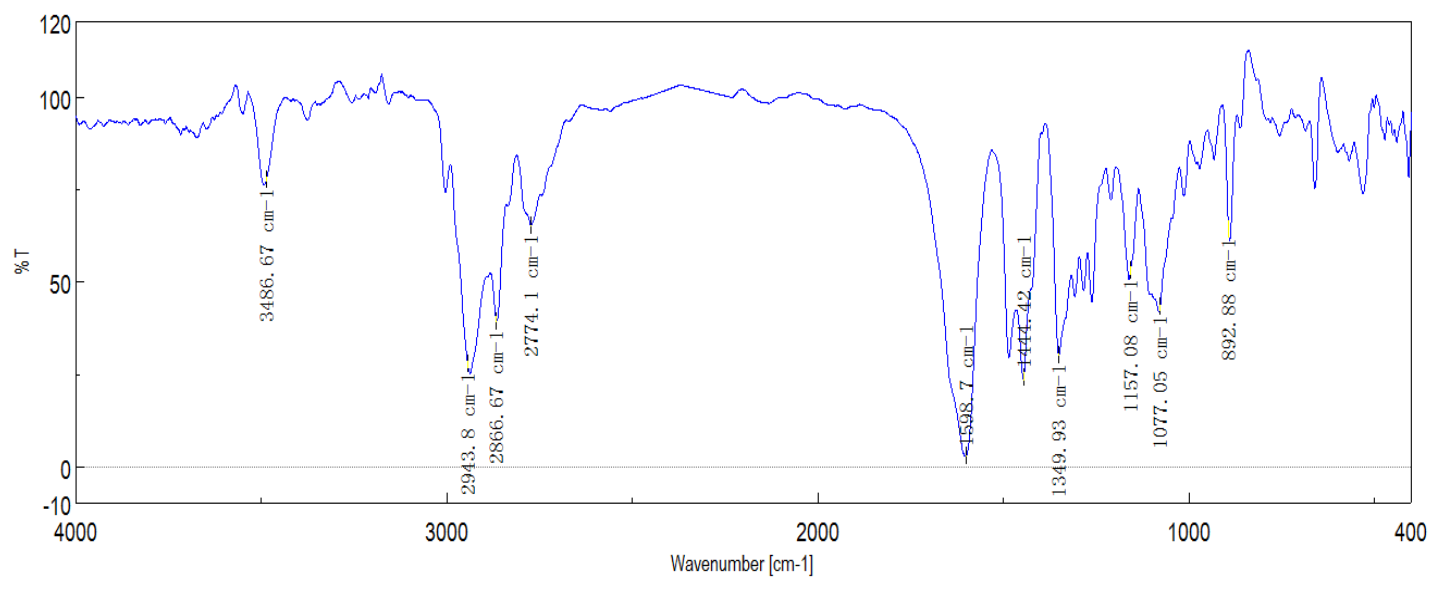

Figure S13. IR spectrum of compound 2

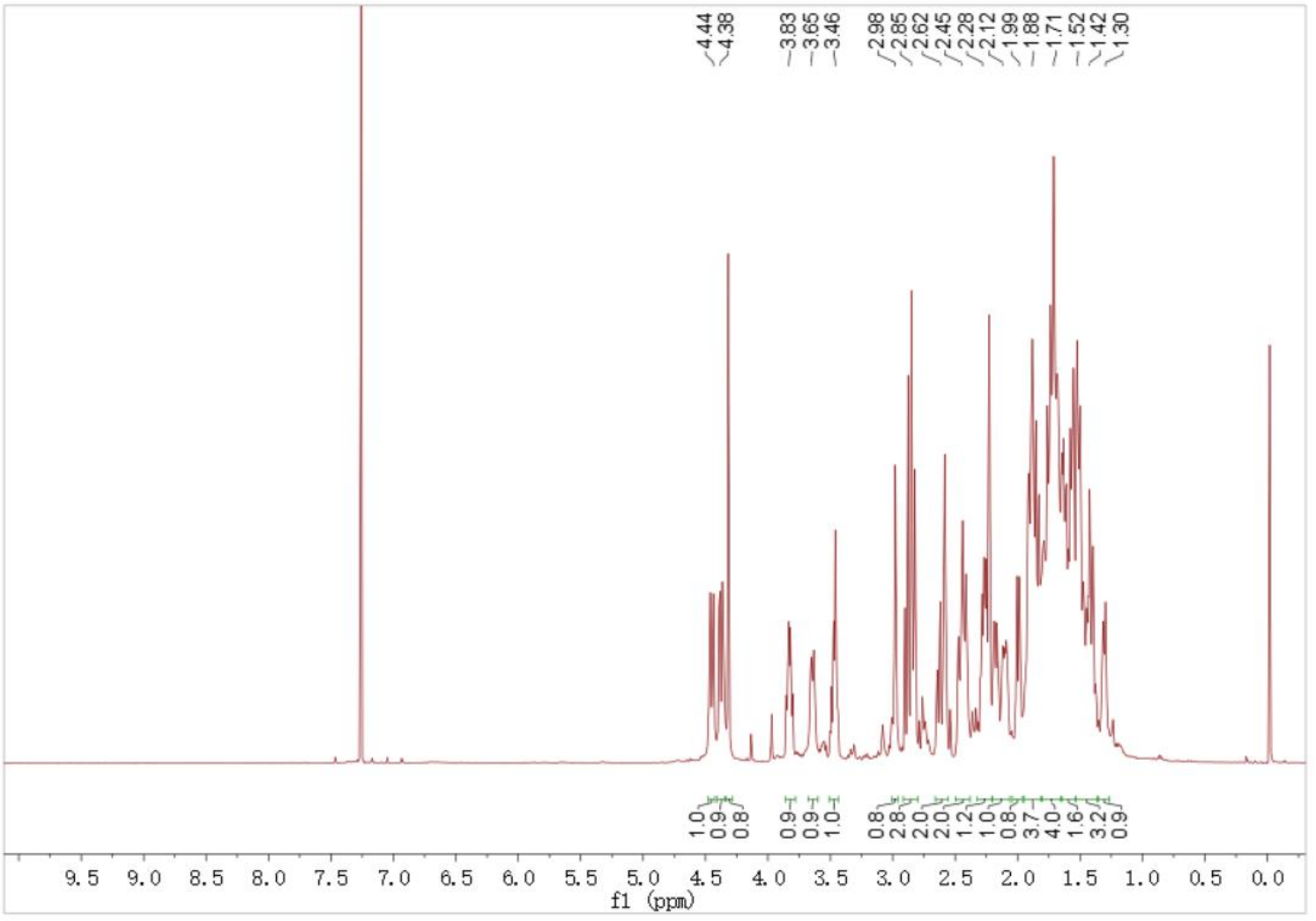

Figure S14. ${ }^{1} \mathrm{H}$ NMR spectrum of compound 2 


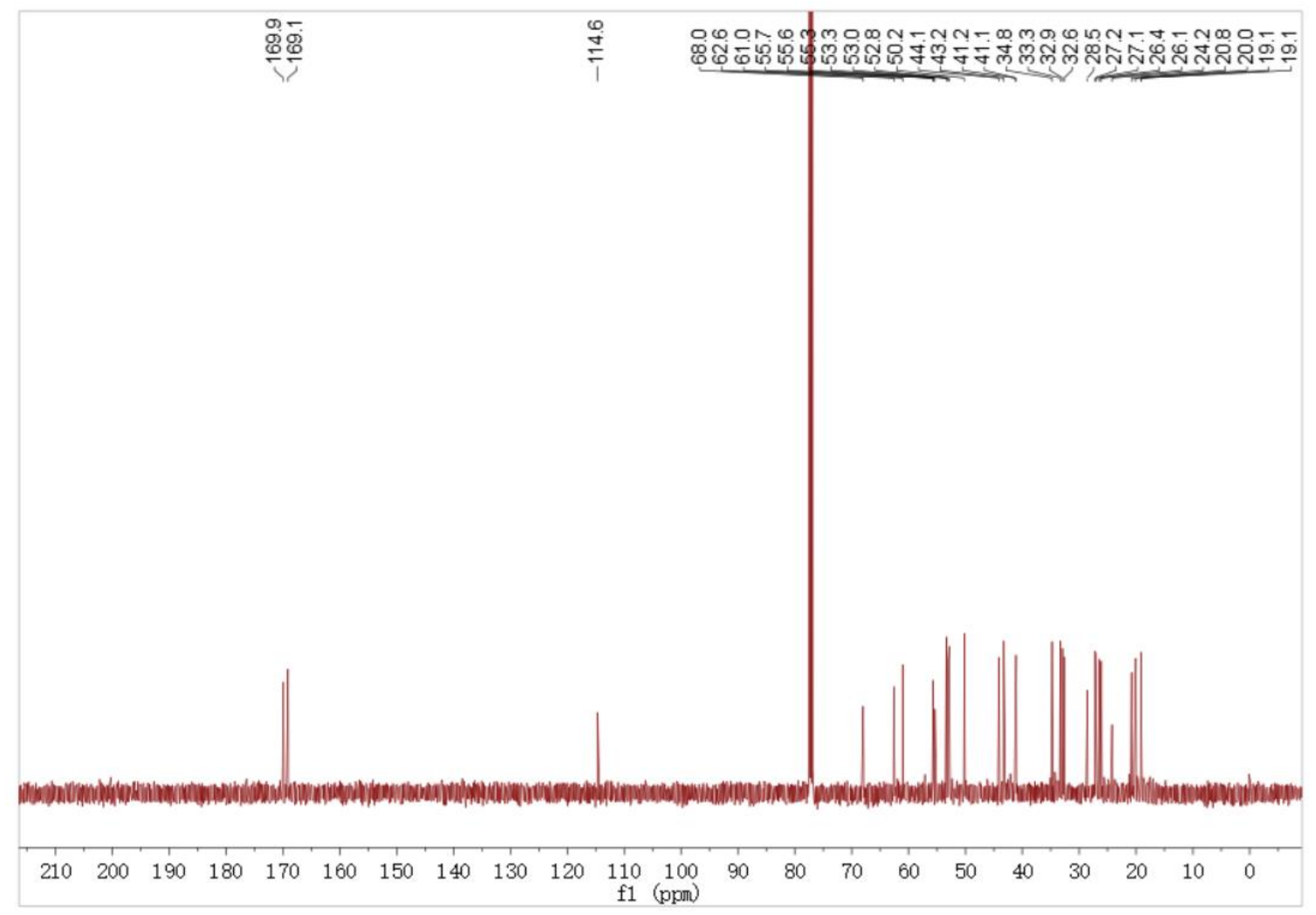

Figure S15. ${ }^{13} \mathrm{C}$ NMR spectrum of compound 2

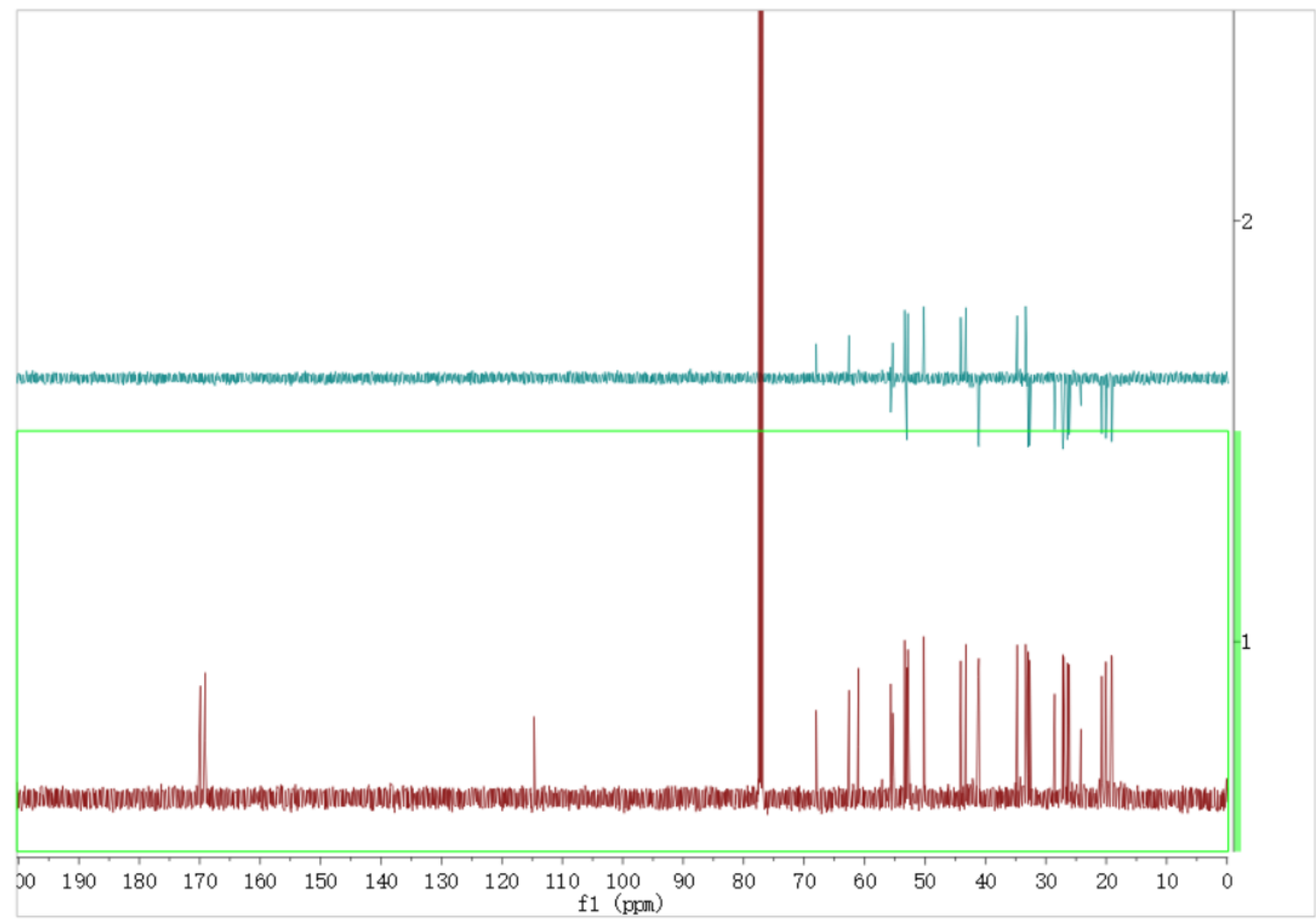

Figure S16. DEPT-135 spectrum of compound 2 


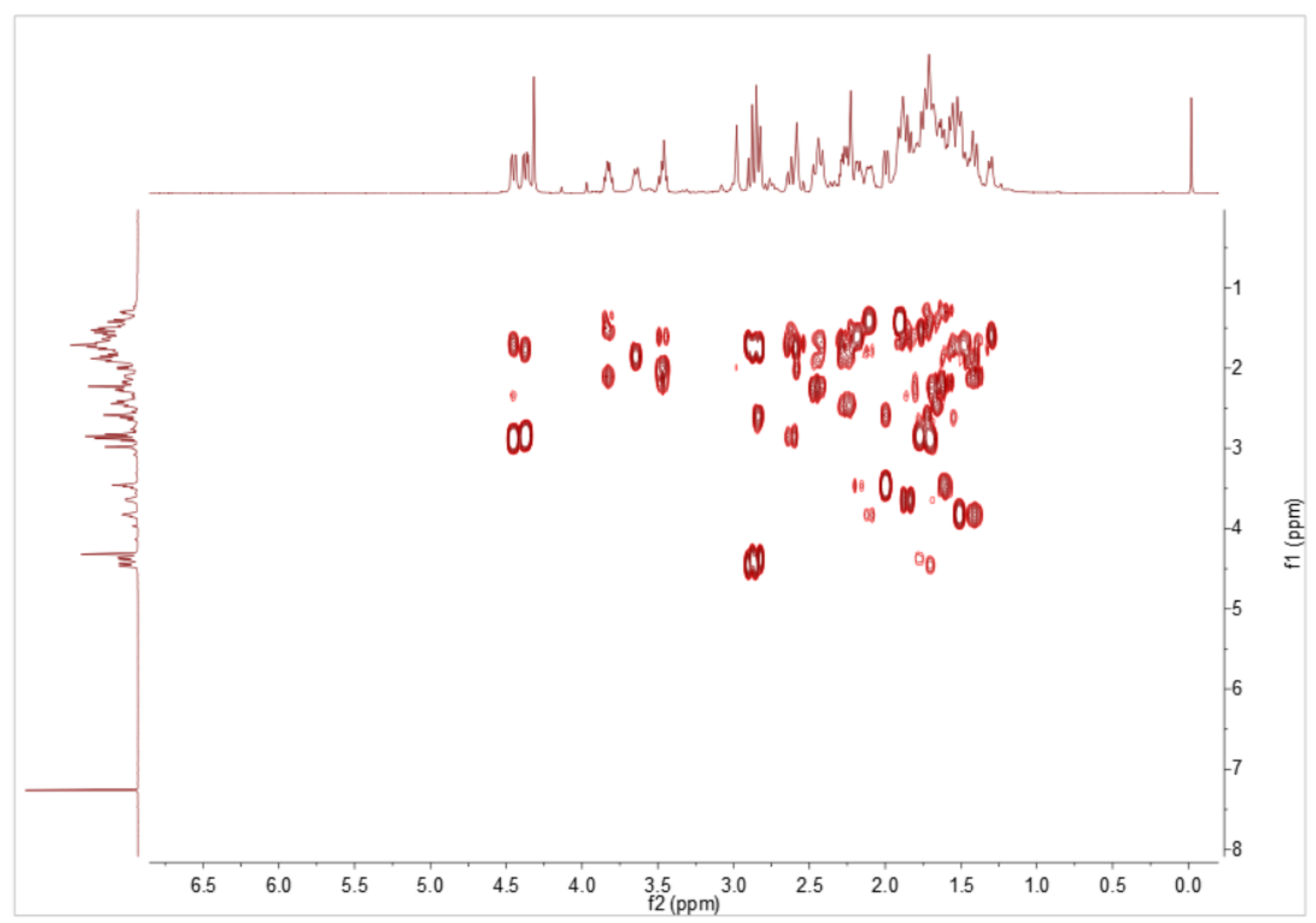

Figure S17. ${ }^{1} \mathrm{H}-{ }^{1} \mathrm{H}$ COSY spectrum of compound 2

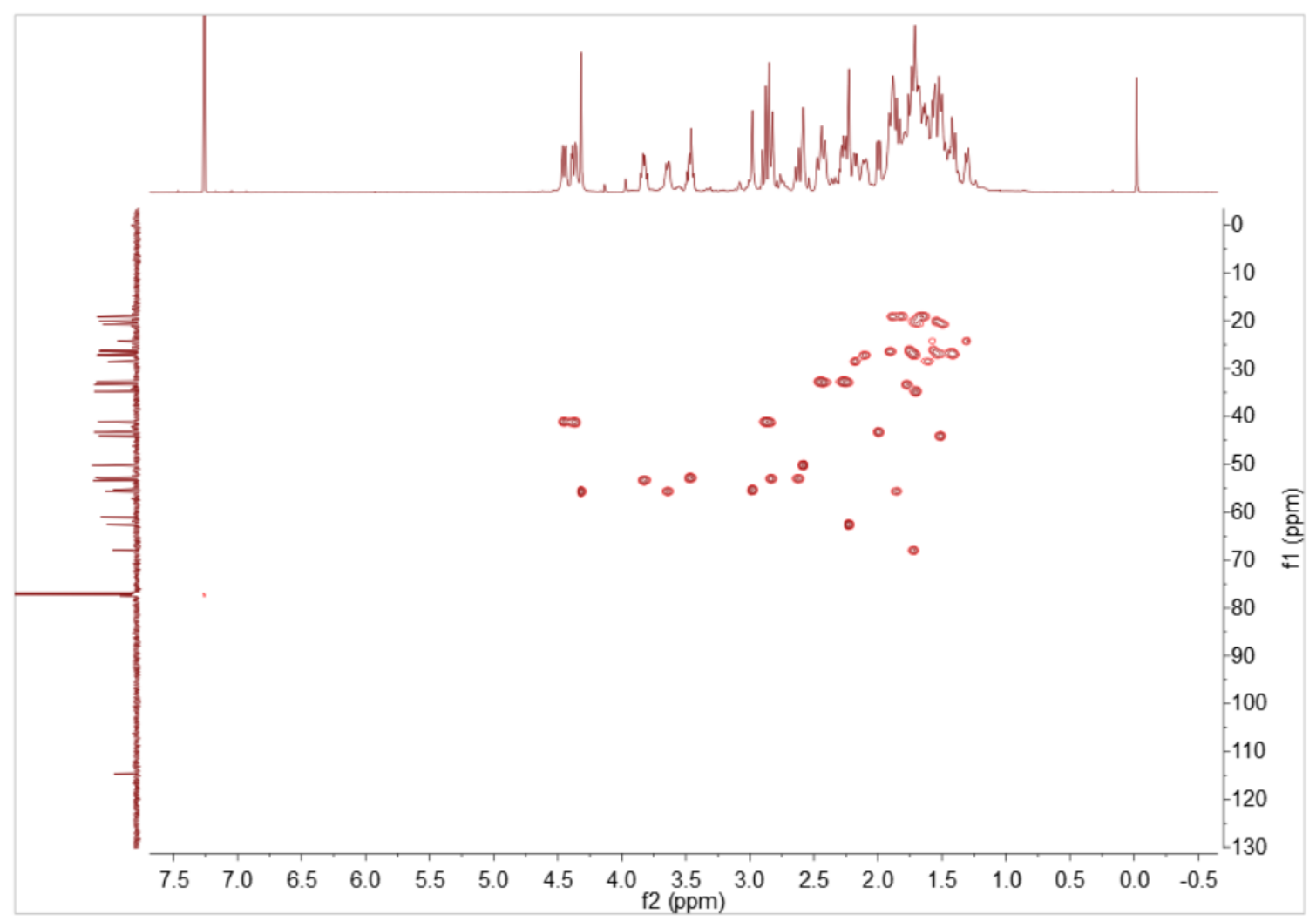

Figure S18. HSQC NMR spectrum of compound 2 


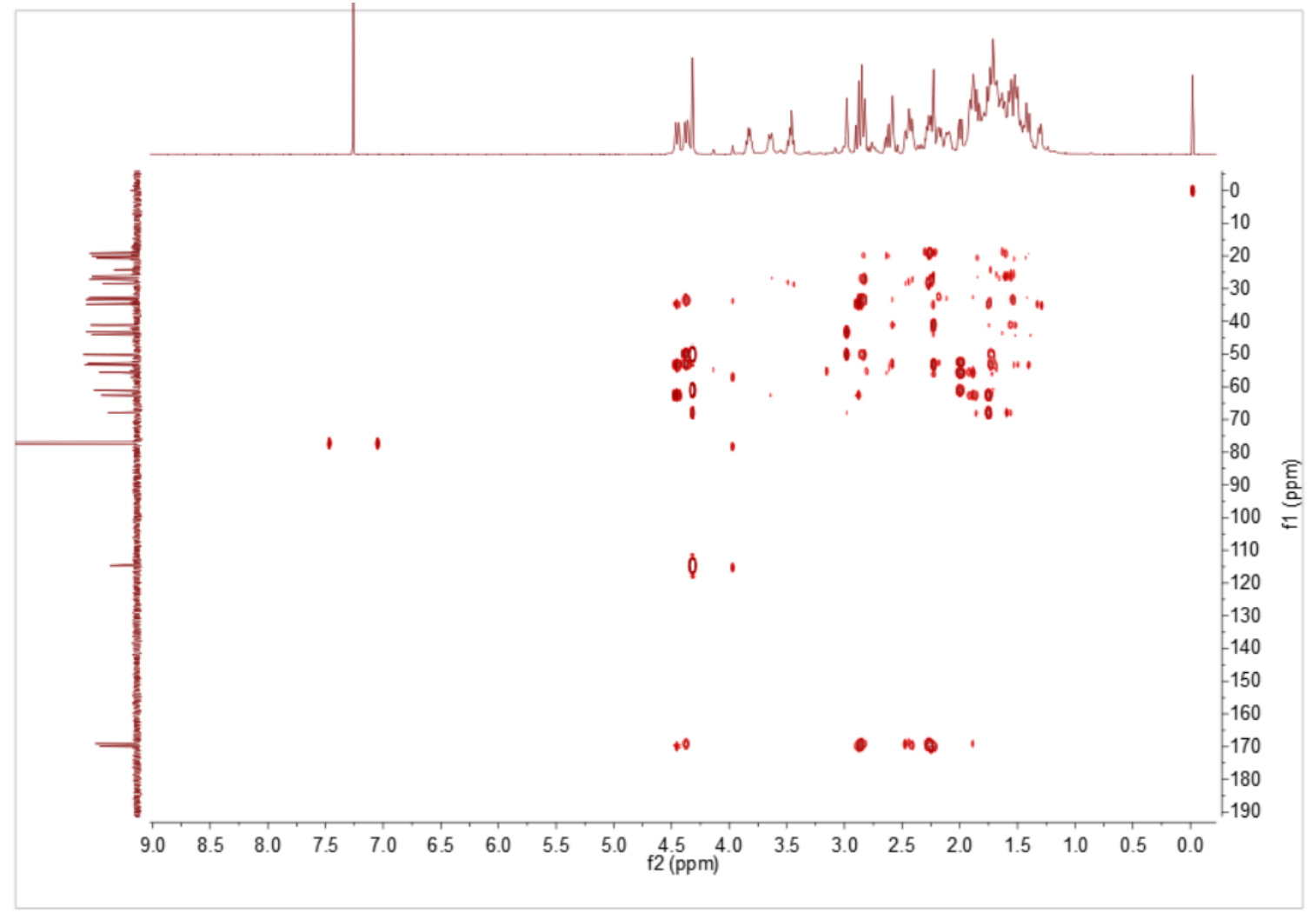

Figure S19. HMBC NMR spectrum of compound 2

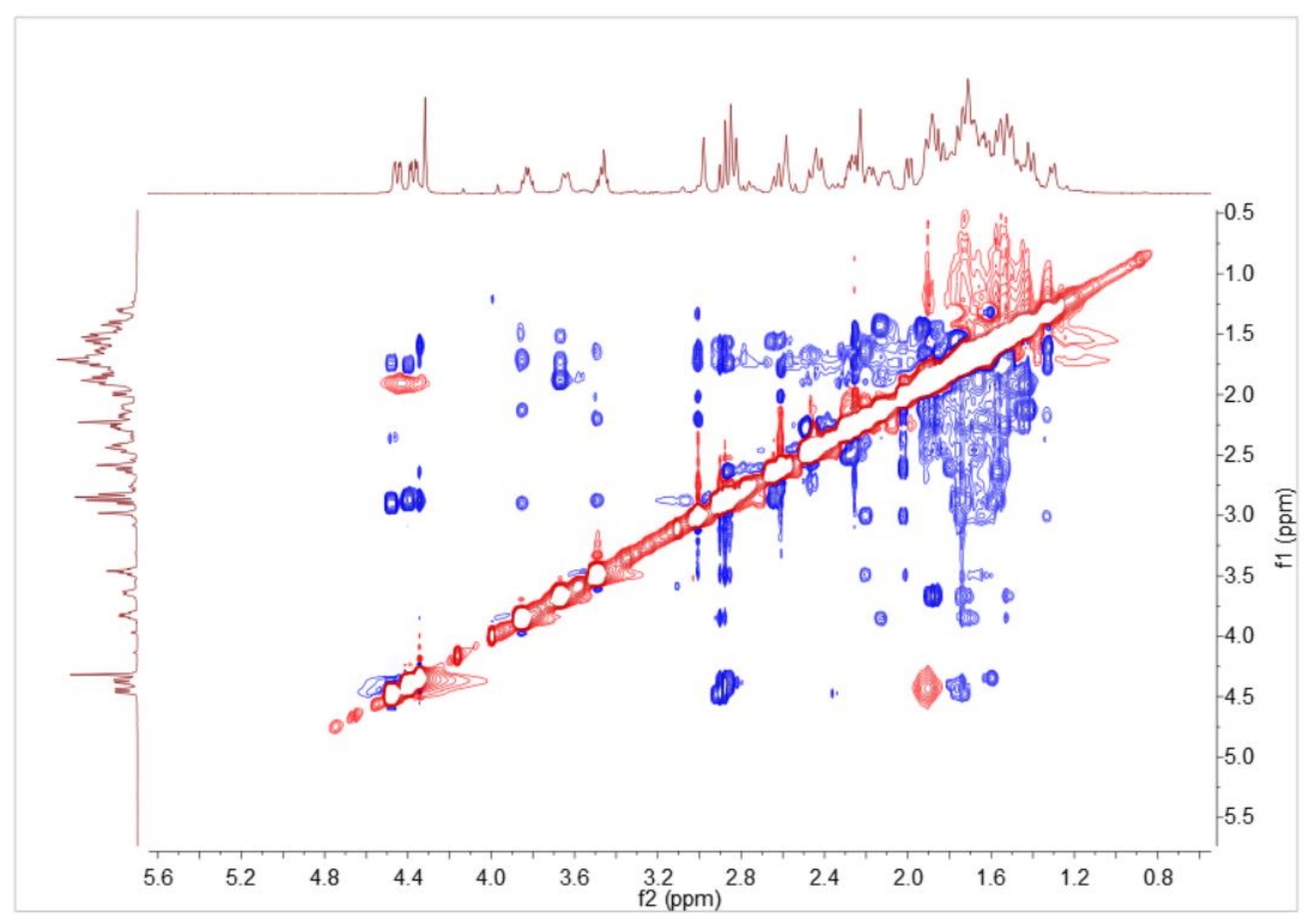

Figure S20. NOESY spectrum of compound 2 


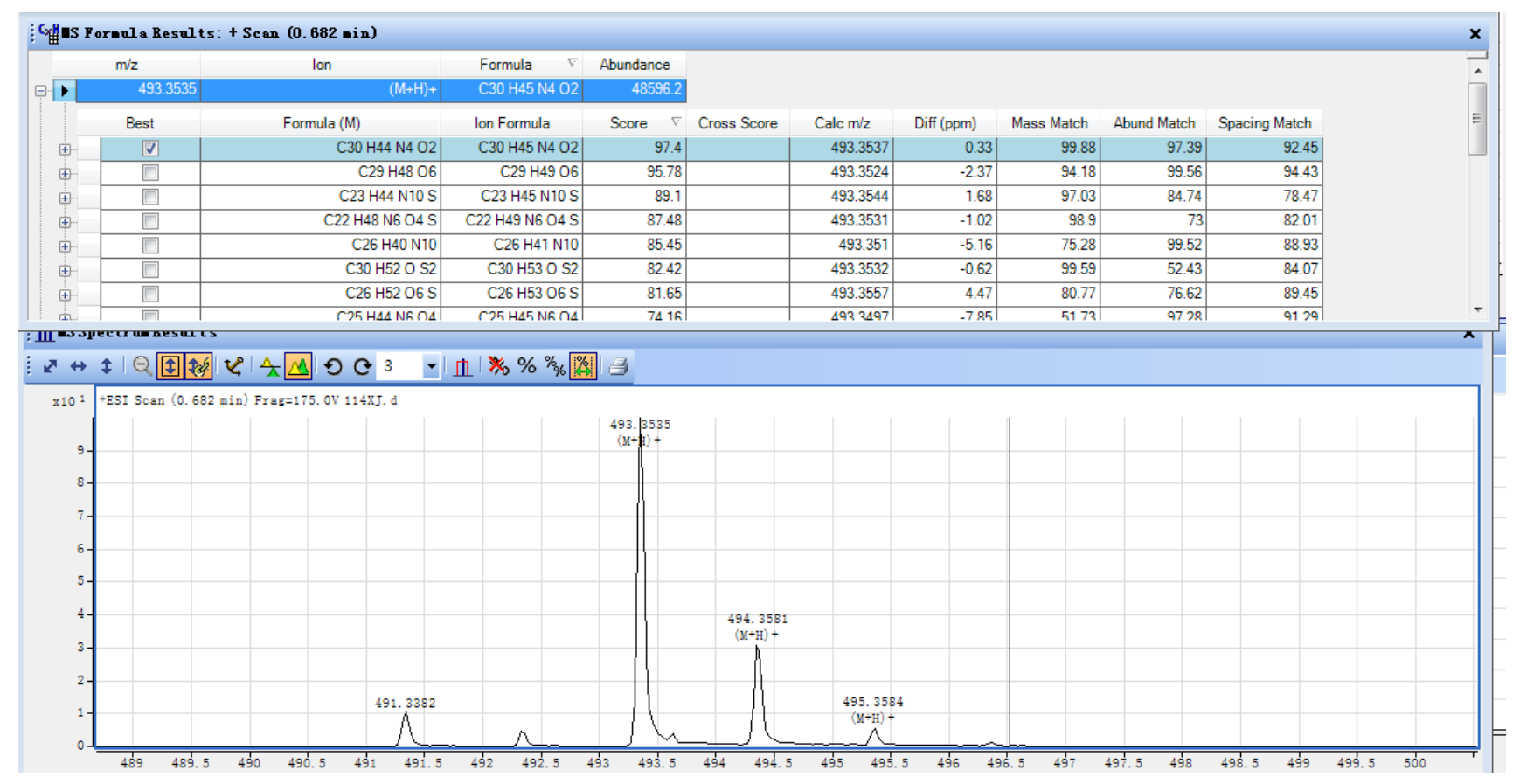

Figure S21. HRESIMS spectrum of compound $\mathbf{3}$

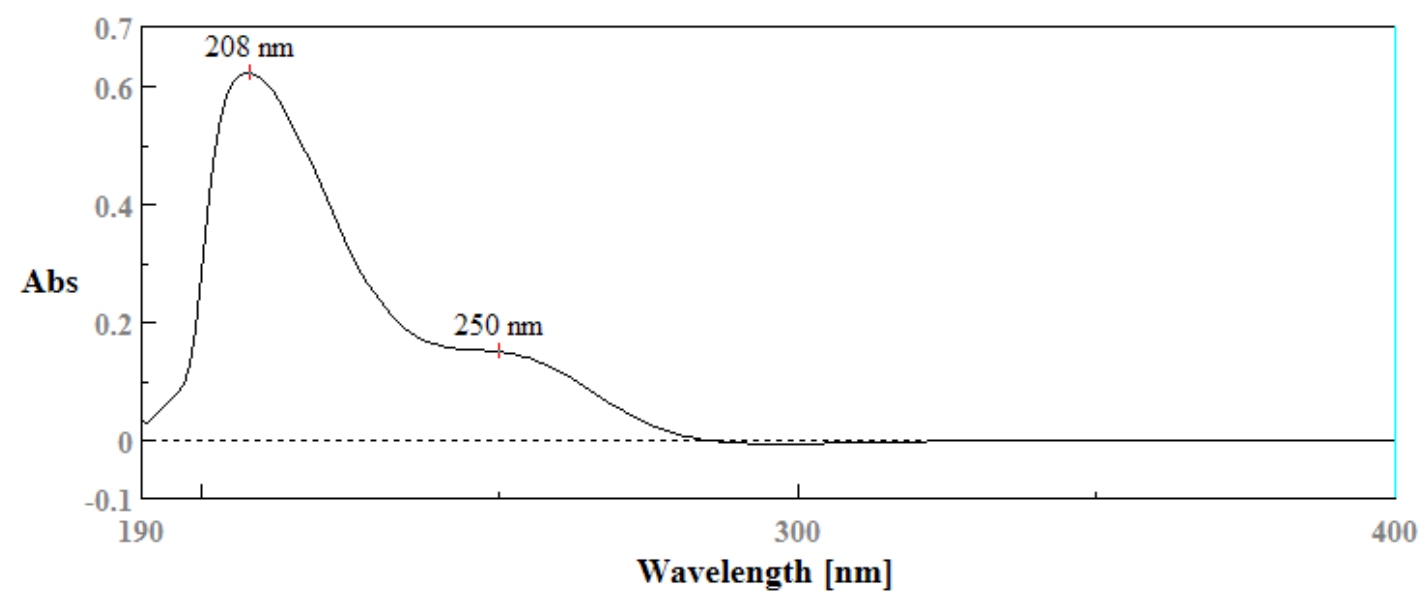

Figure S22. UV spectrum of compound 3 


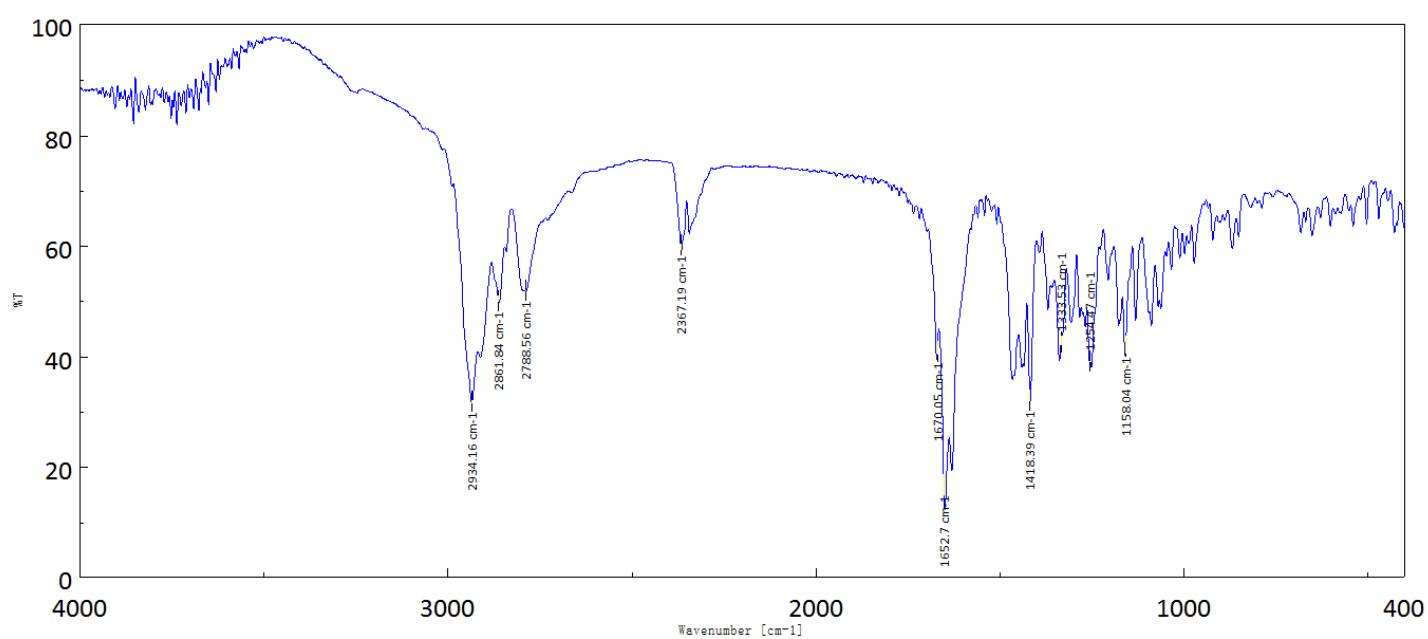

Figure S23. IR spectrum of compound 3

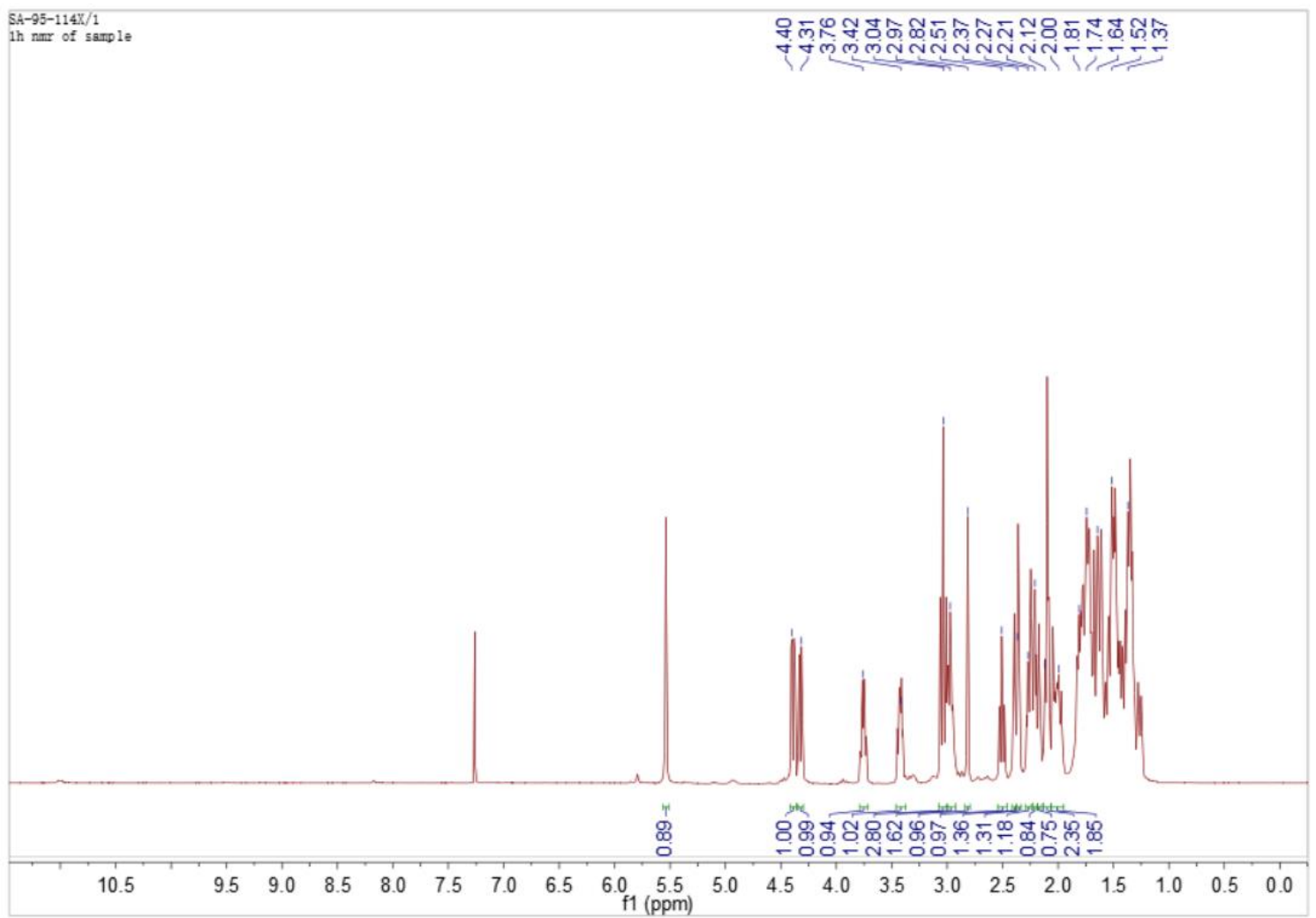

Figure S24. ${ }^{1} \mathrm{H}$ NMR spectrum of compound 3 


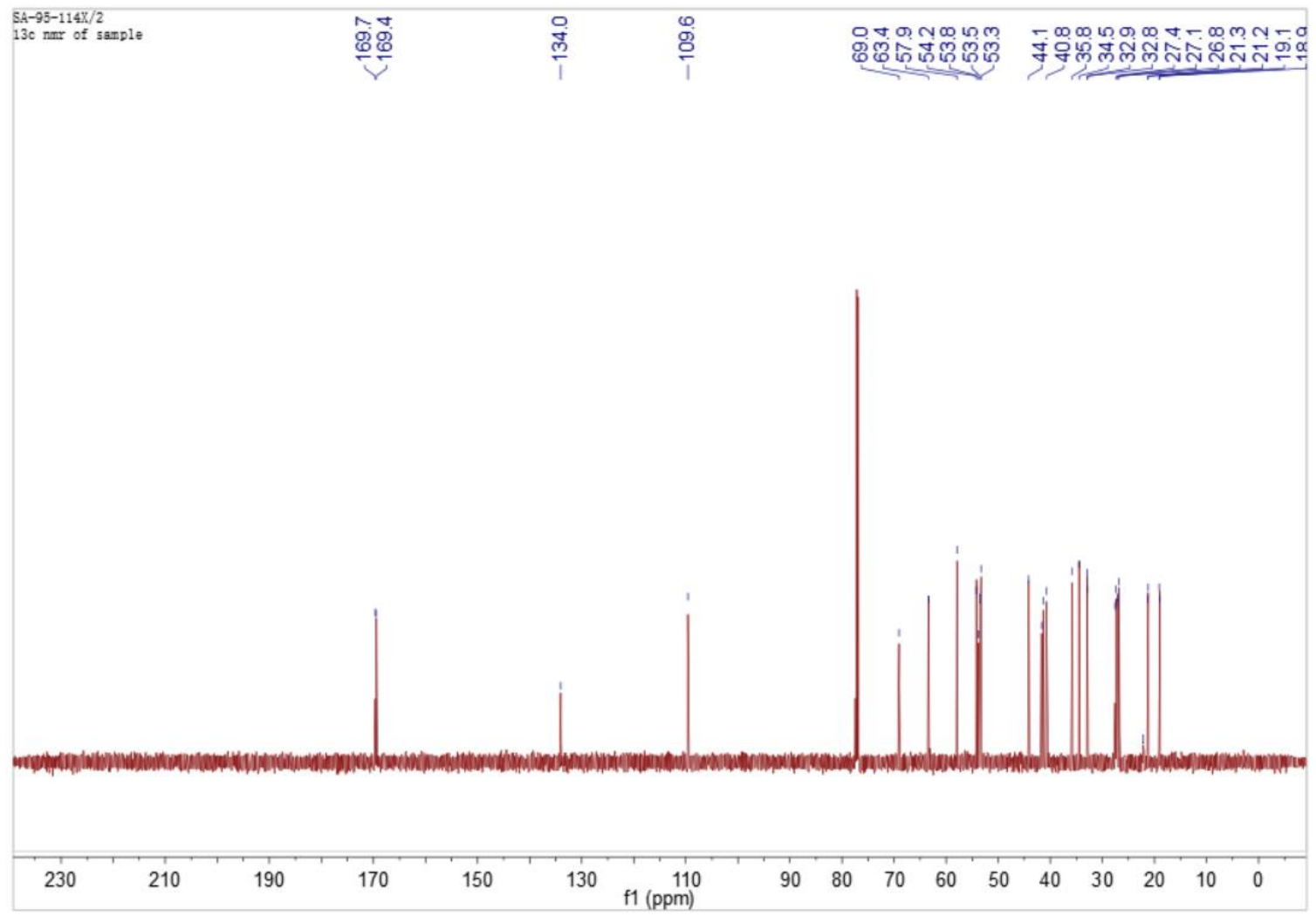

Figure S25. ${ }^{13} \mathrm{C}$ NMRspectrum of compound $\mathbf{3}$

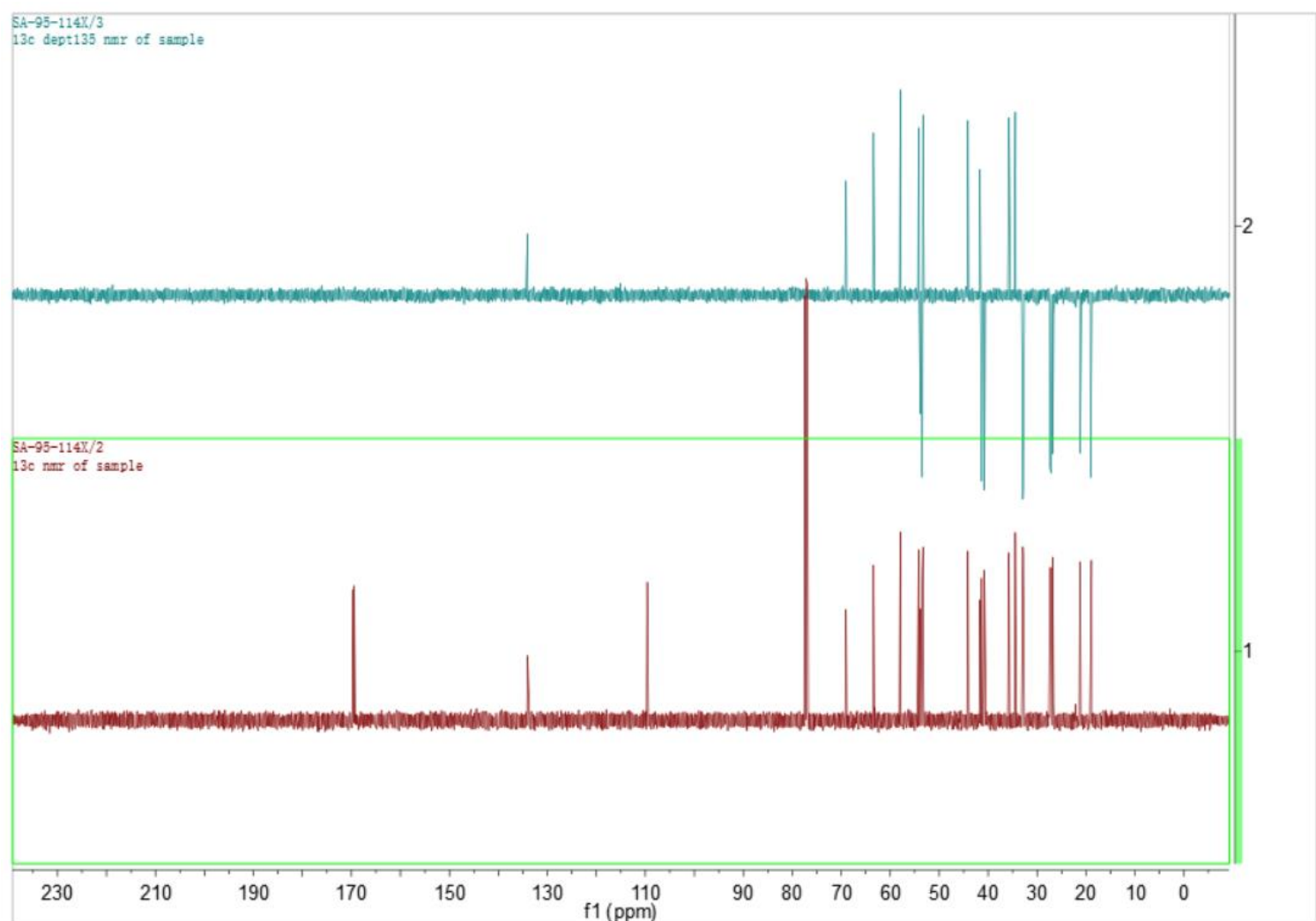

Figure S26. DEPT-135 spectrum of compound $\mathbf{3}$ 


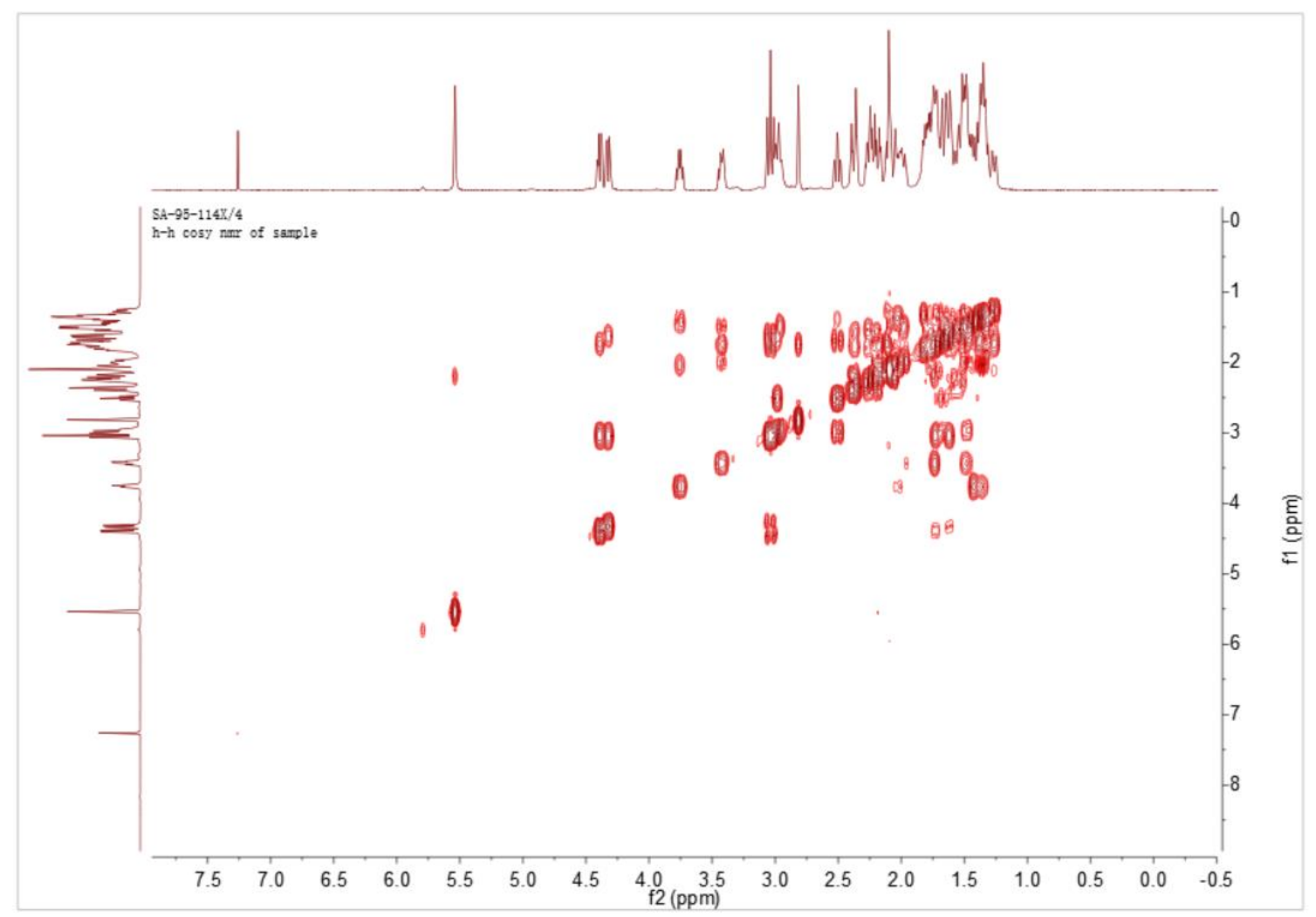

Figure S27. ${ }^{1} \mathrm{H}-{ }^{1} \mathrm{H}$ COSY spectrum of compound $\mathbf{3}$

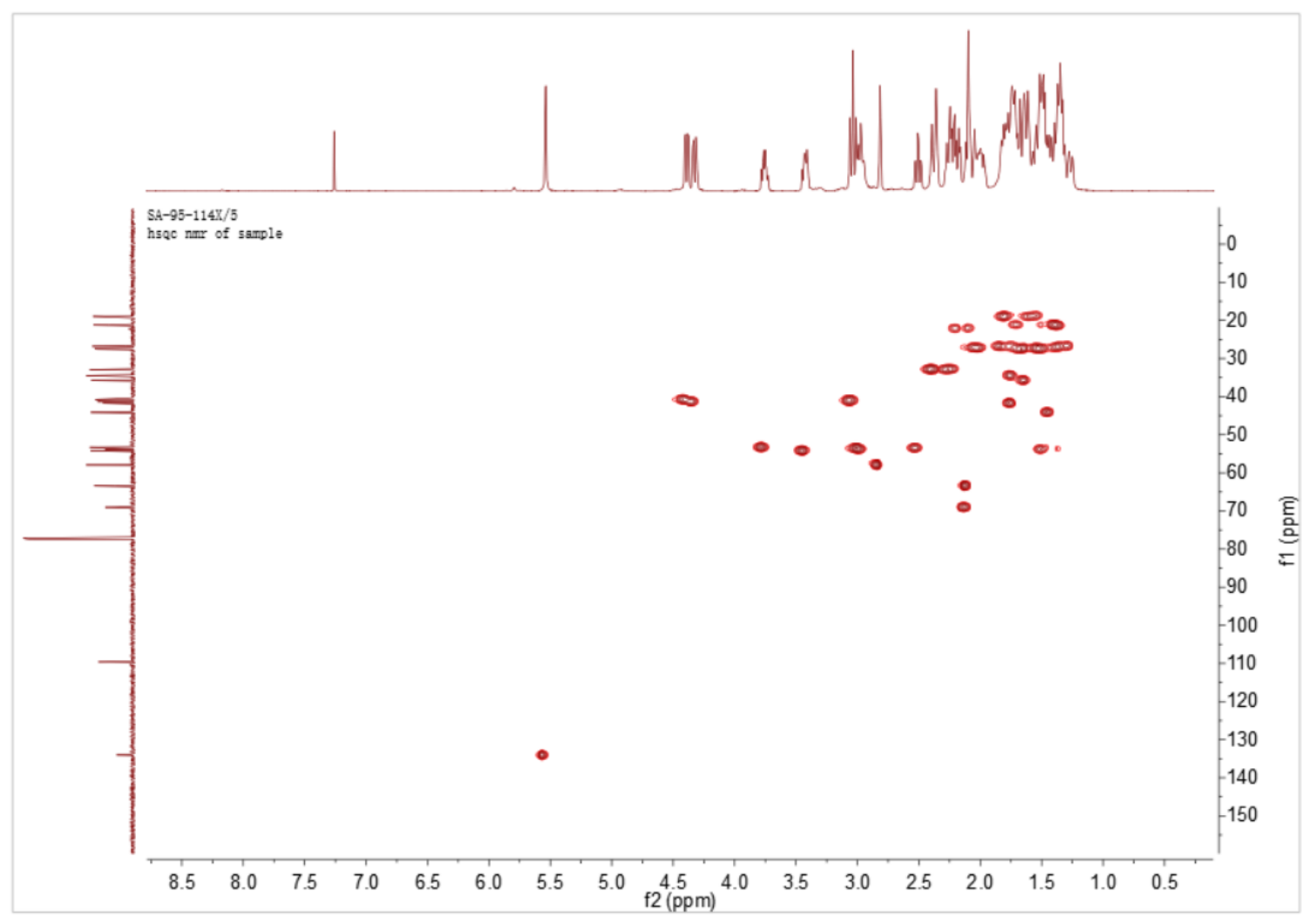

Figure S28. HSQC NMR spectrum of compound 3 


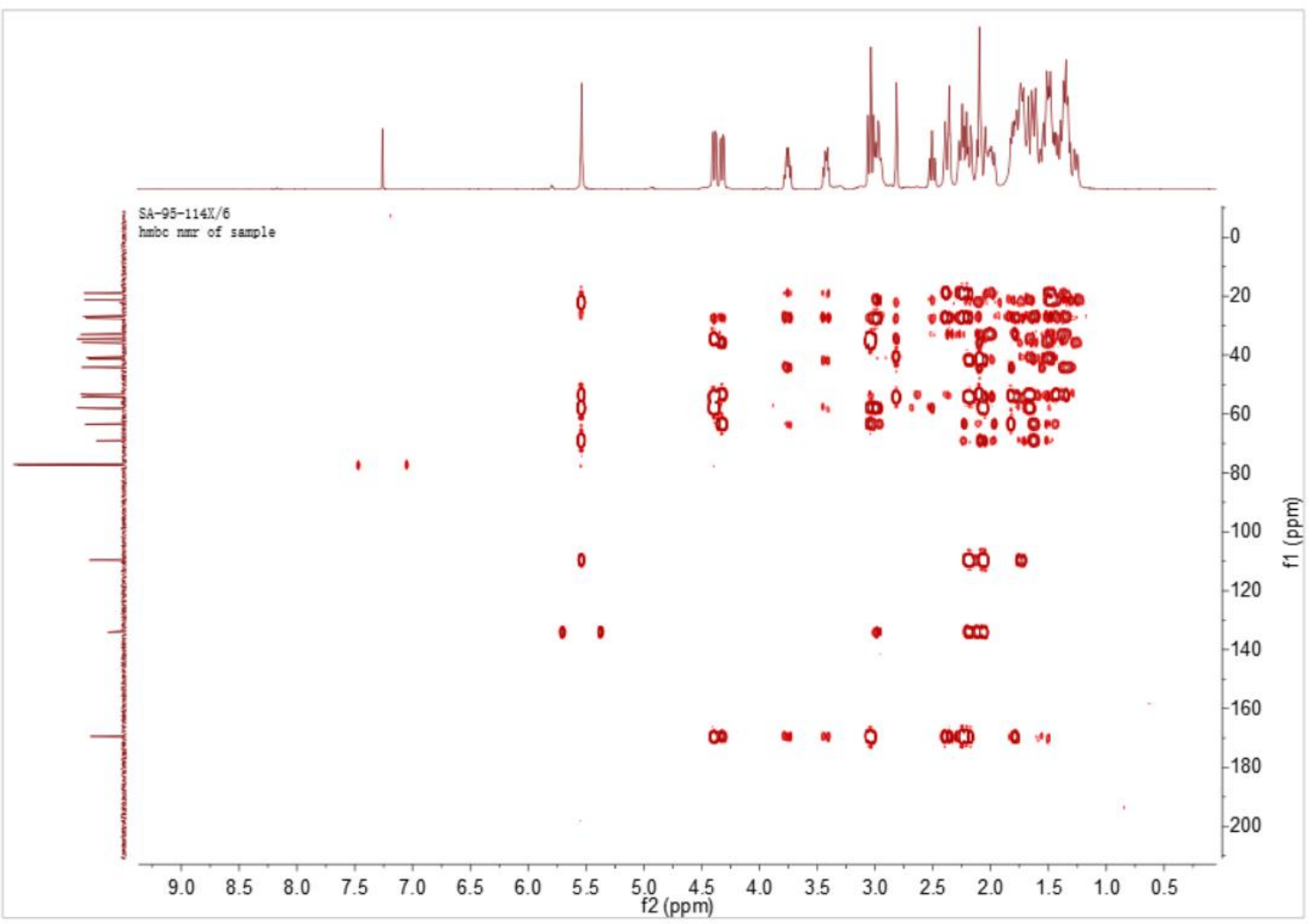

Figure S29. HMBC NMR spectrum of compound 3

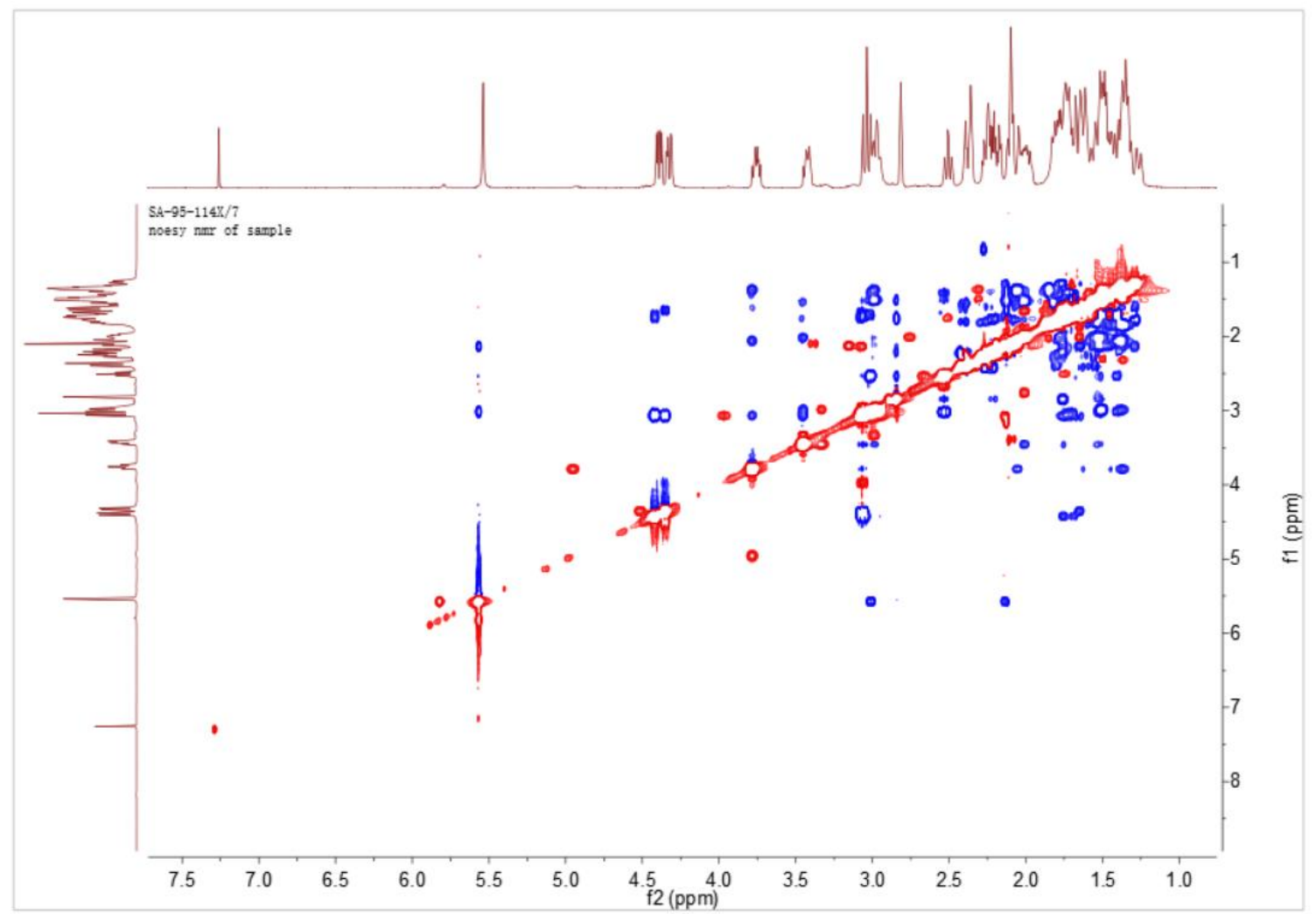

Figure S30. NOESY spectrum of compound $\mathbf{3}$ 


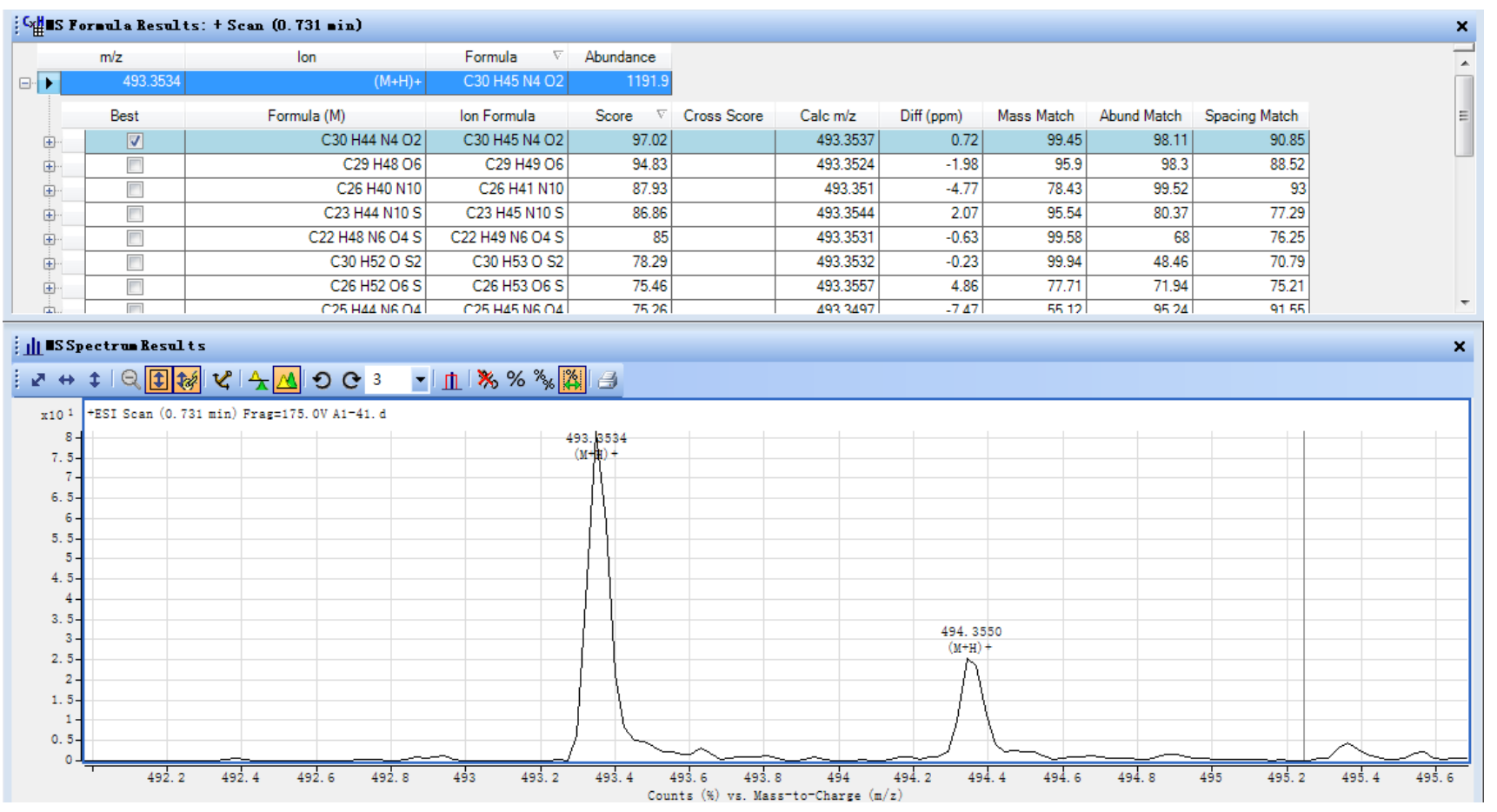

Figure S31. HRESIMS spectrum of compound 4

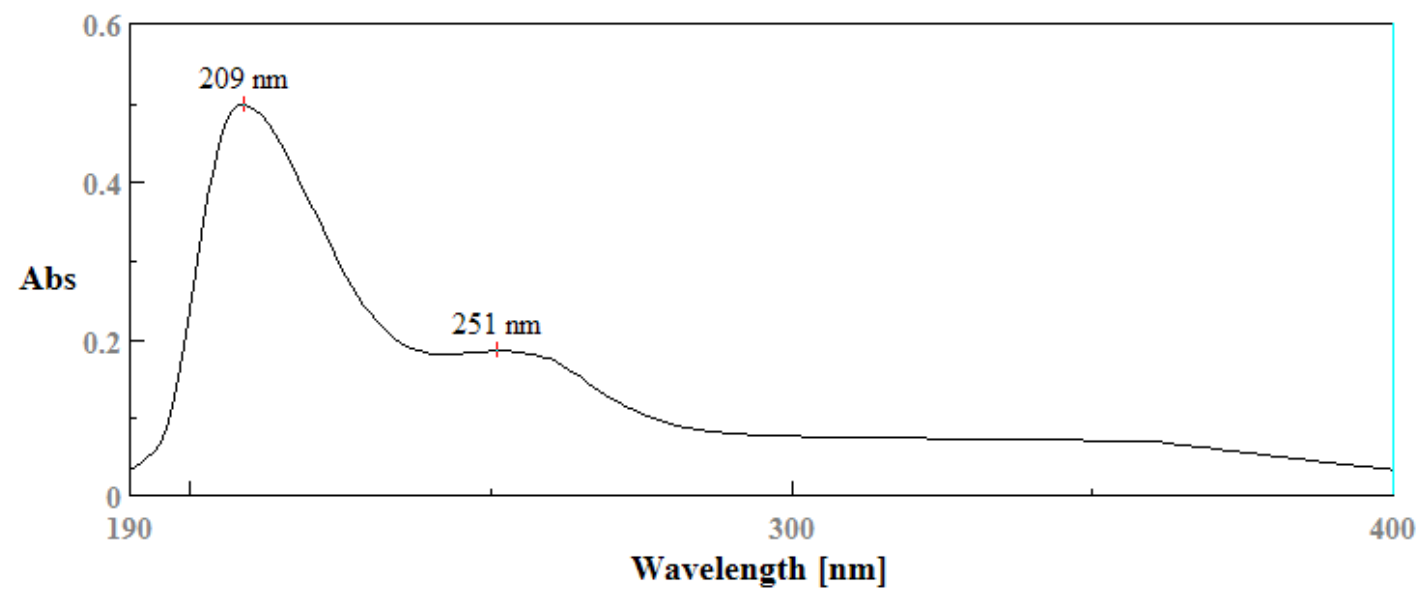

Figure S32. UV spectrum of compound 4 


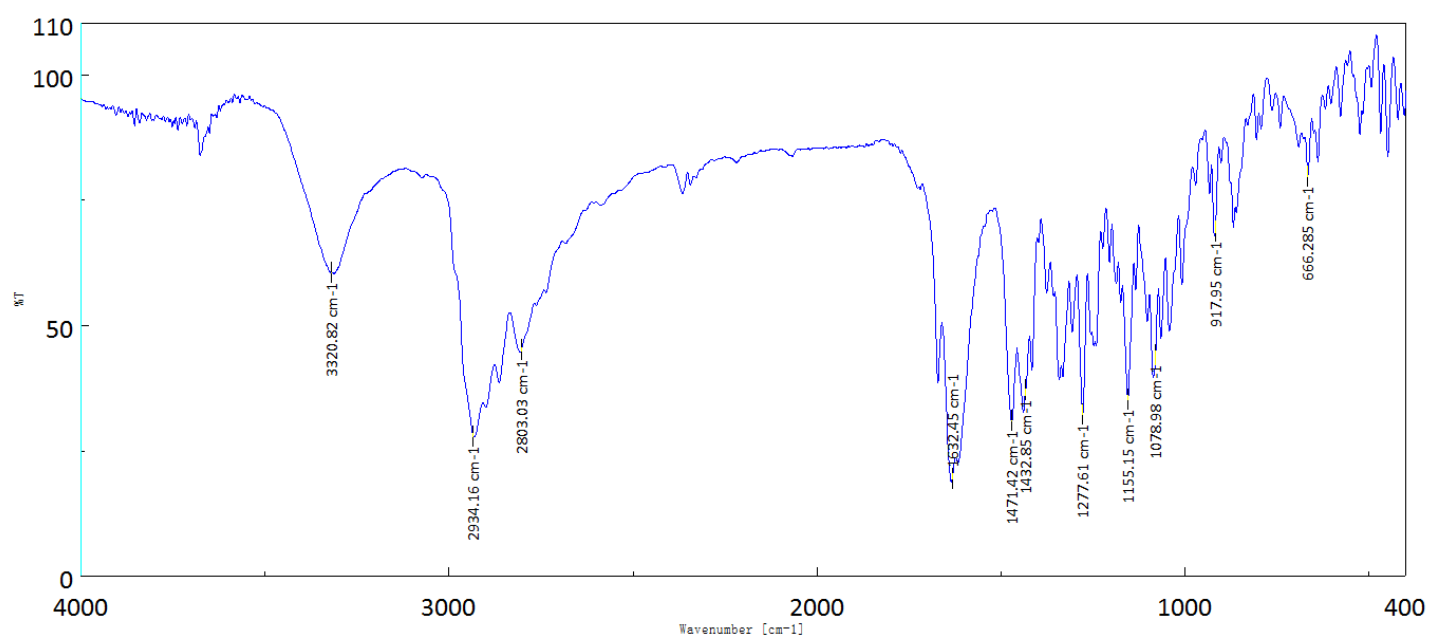

Figure S33. IR spectrum of compound 4

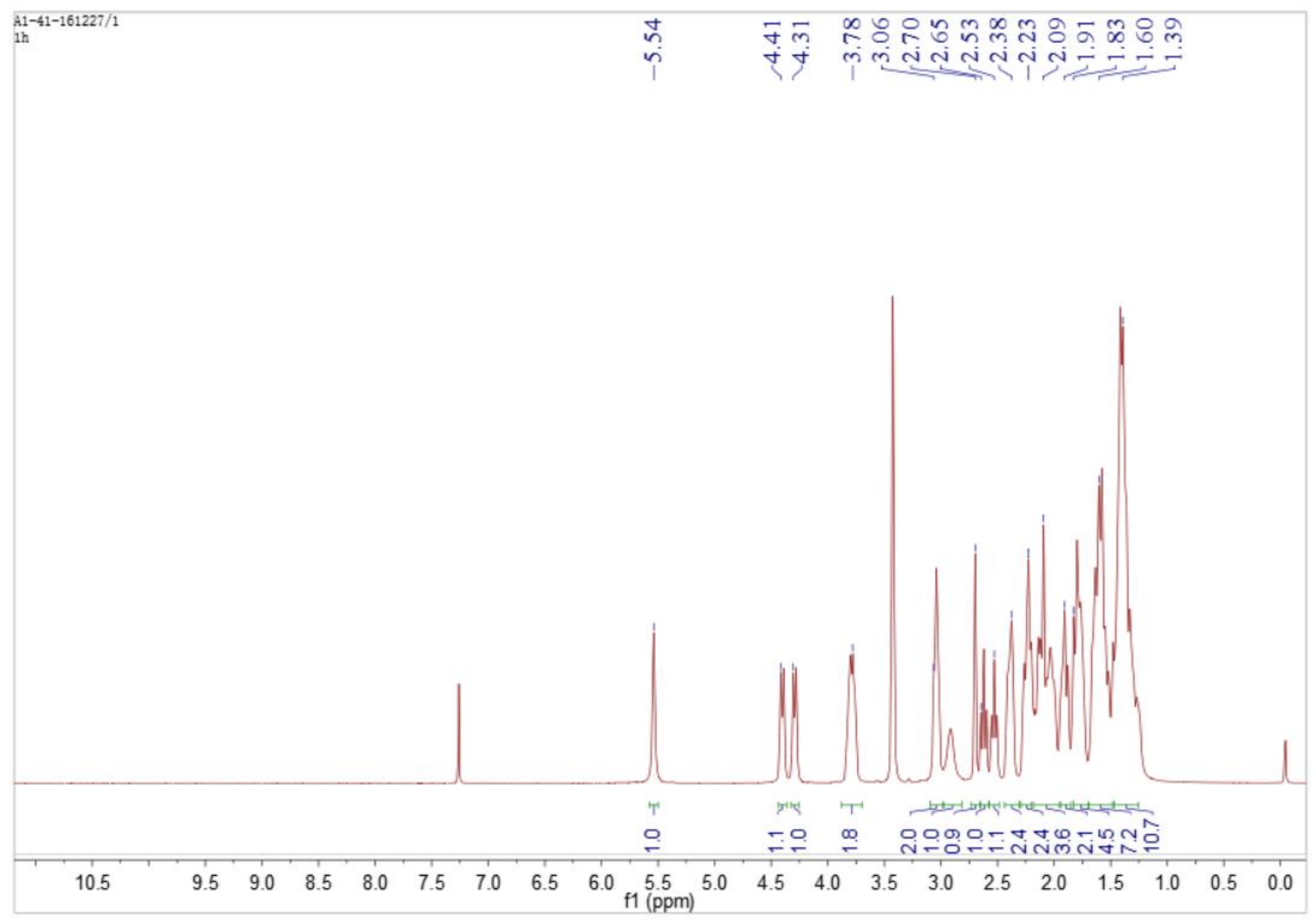

Figure S34. ${ }^{1} \mathrm{H}$ NMR spectrum of compound 4 


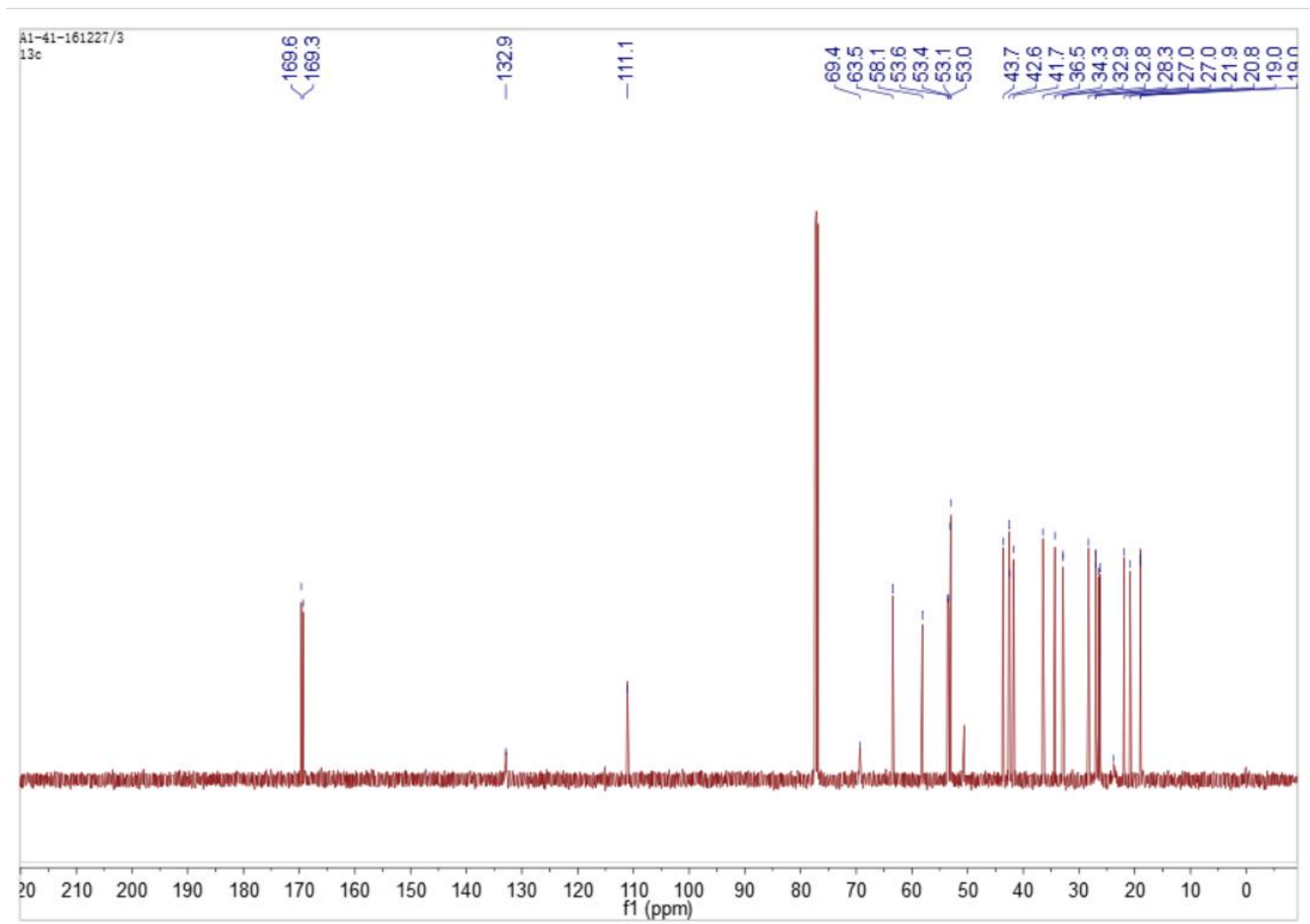

Figure S35. ${ }^{13} \mathrm{C}$ NMR spectrum of compound 4

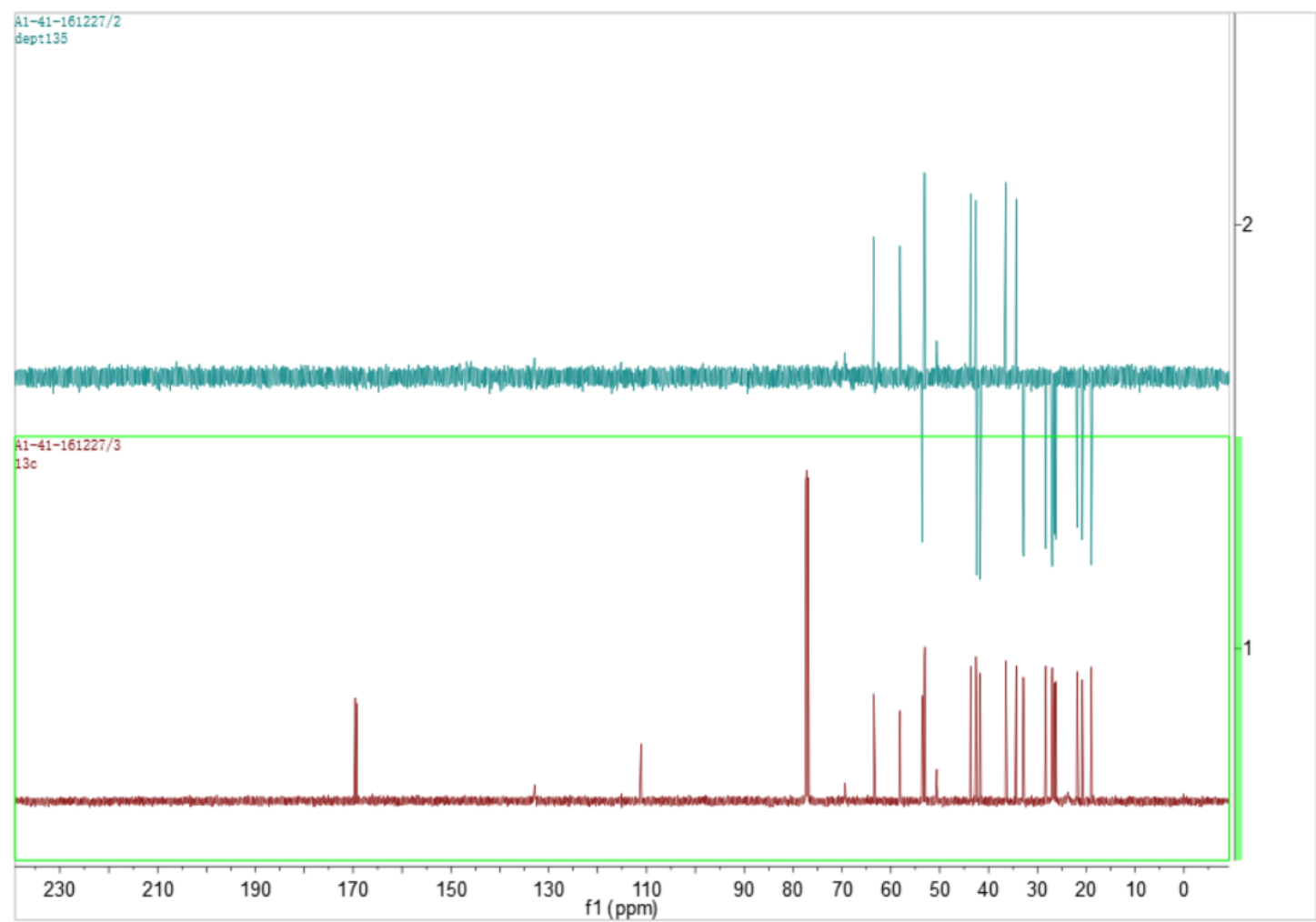

Figure S36. DEPT-135 spectrum of compound 4 


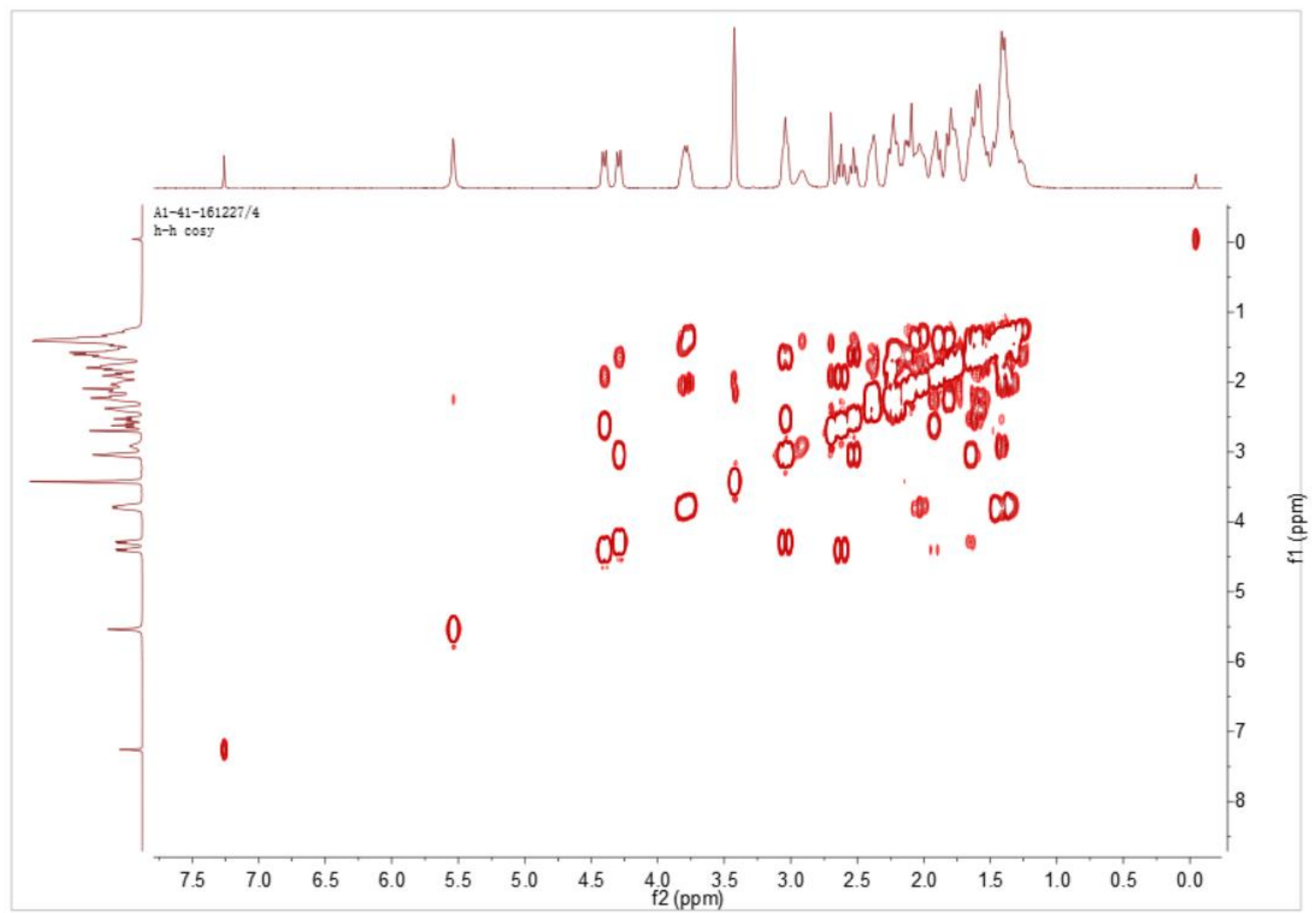

Figure S37. ${ }^{1} \mathrm{H}-{ }^{1} \mathrm{H}$ COSY spectrum of compound 4

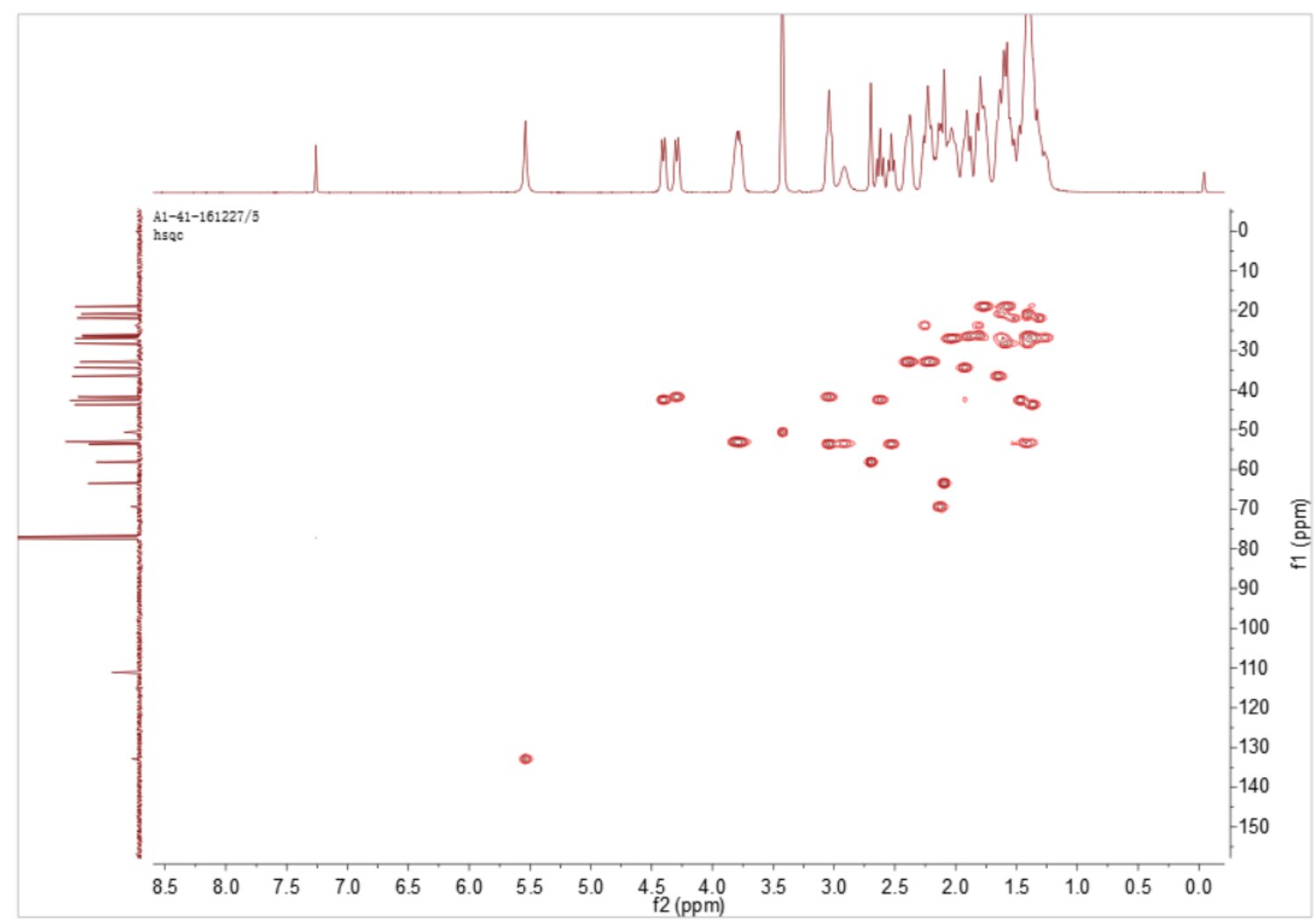

Figure S38. HSQC NMR spectrum of compound 4 


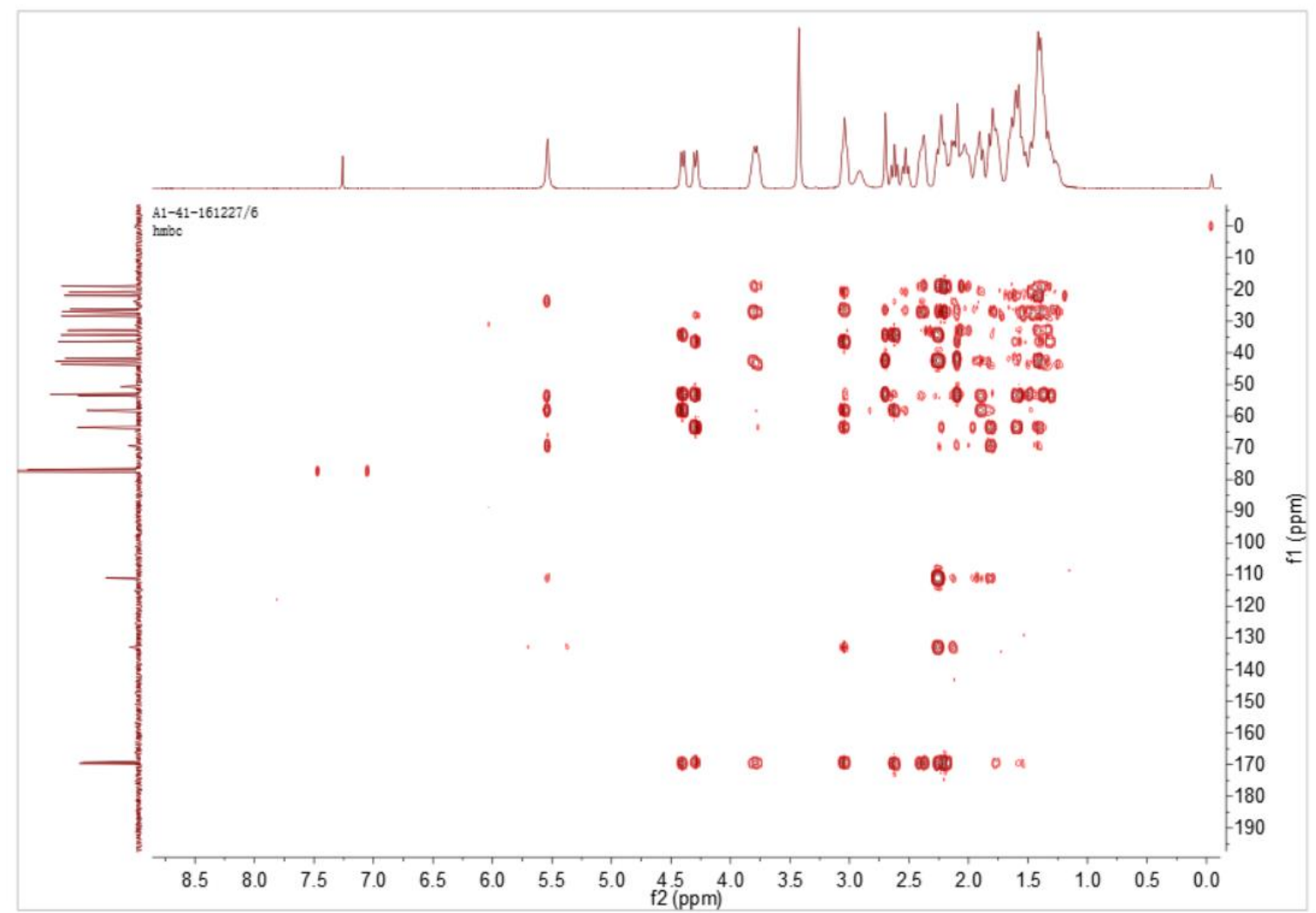

Figure S39. HMBC NMR spectrum of compound 4

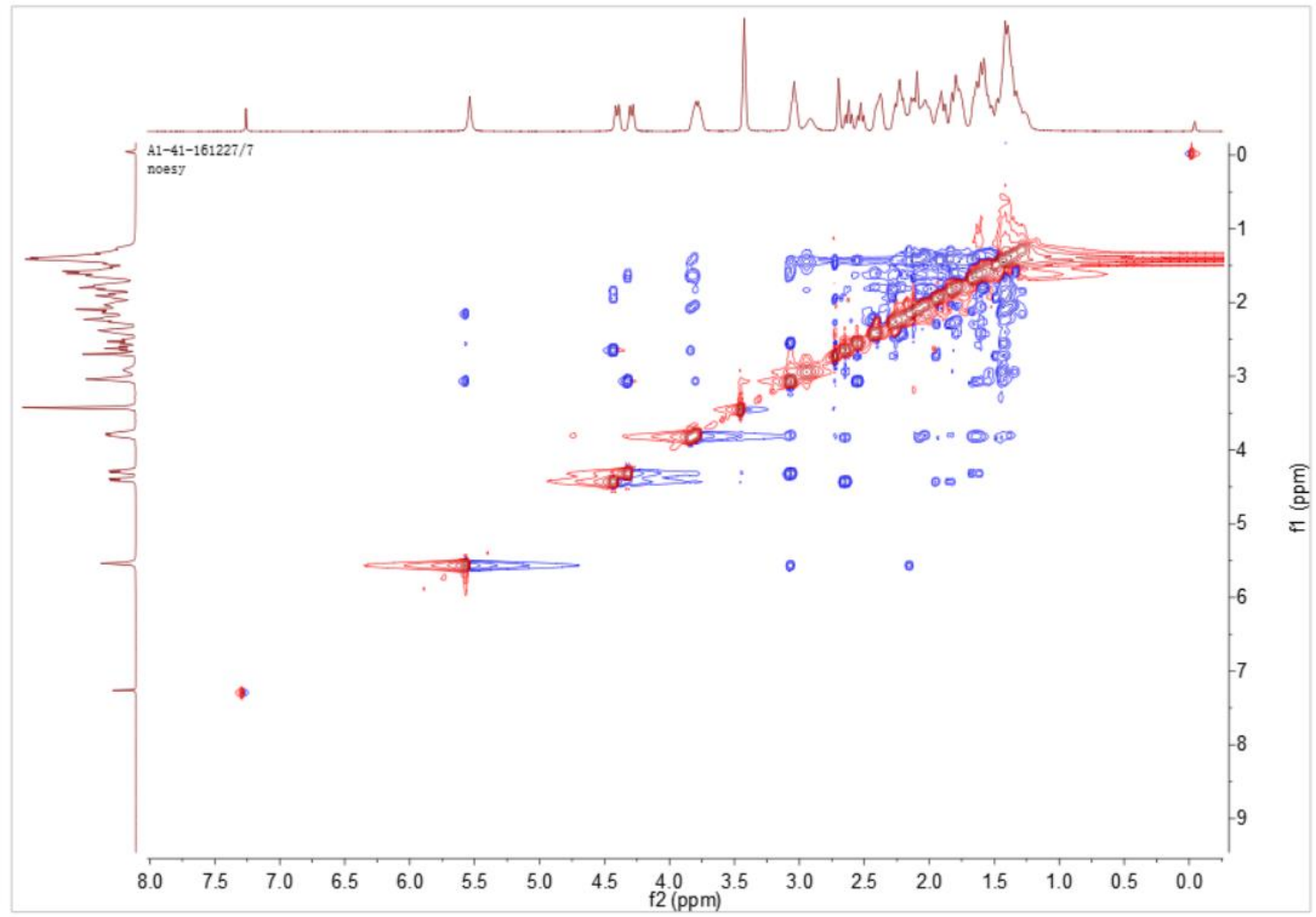

Figure S40. NOESY spectrum of compound 4 


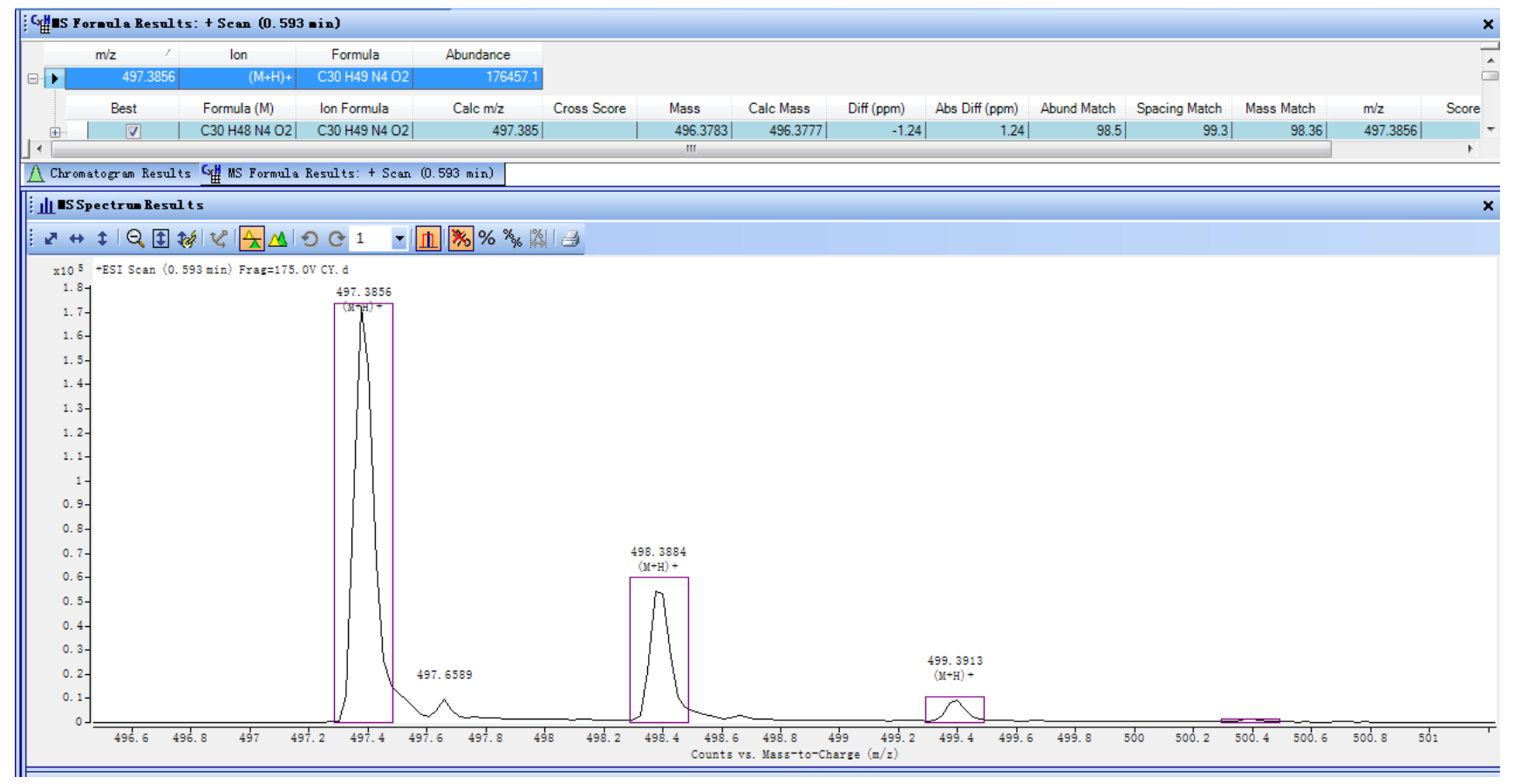

Figure S41. HRESIMS spectrum of compound 5

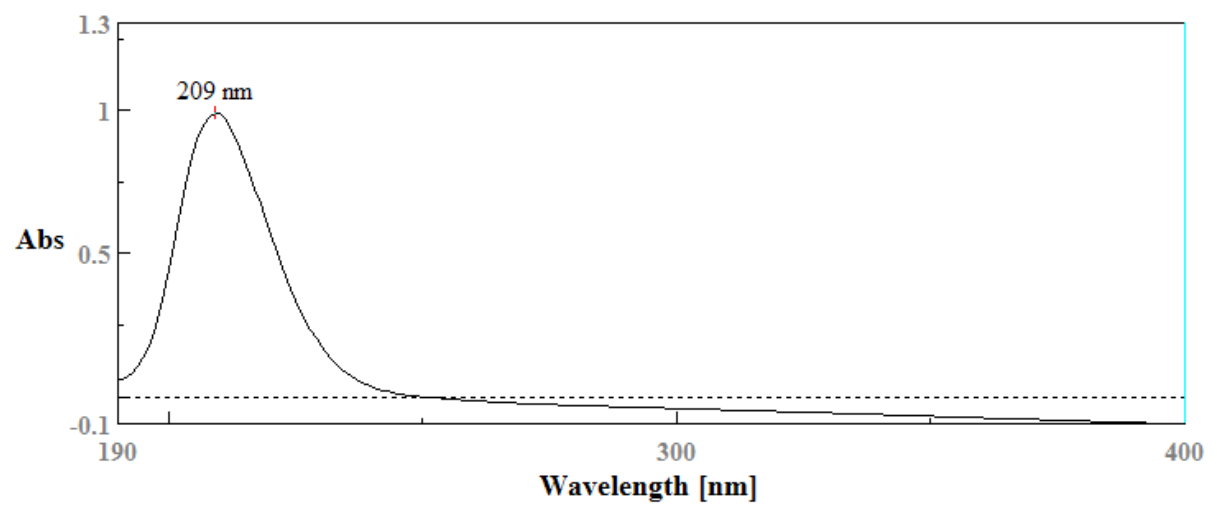

Figure S42. UV spectrum of compound 5

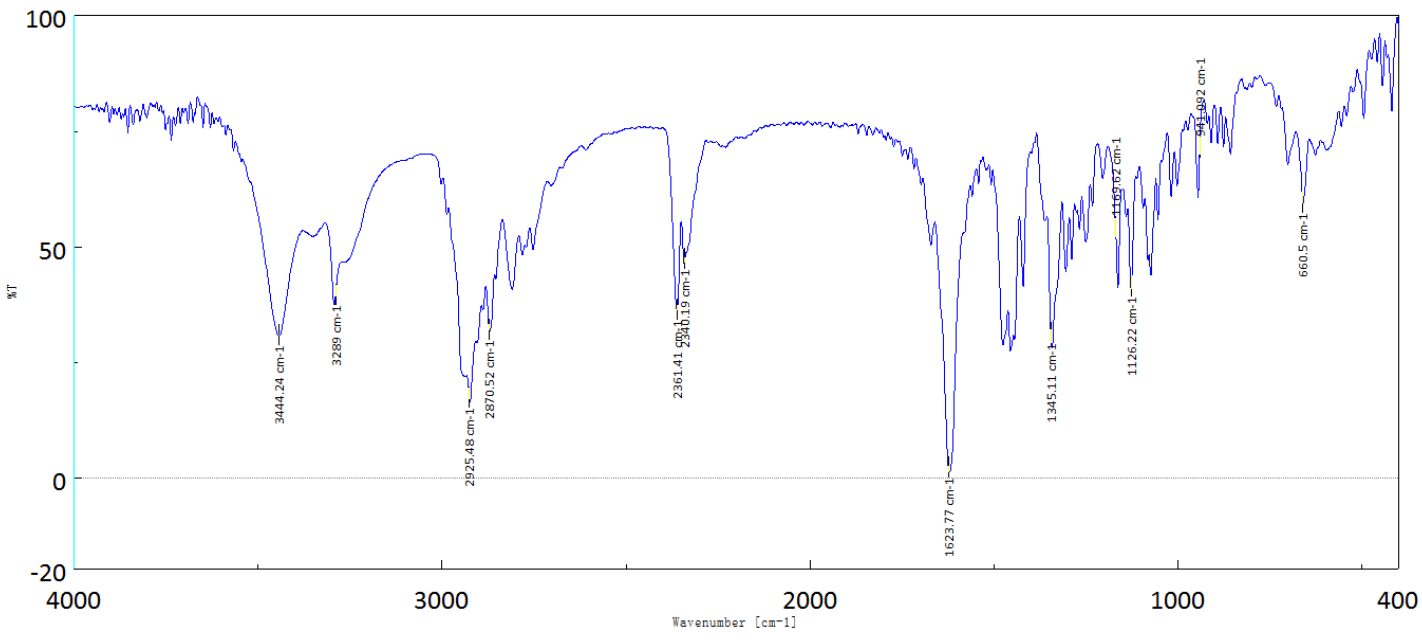

Figure S43. IR spectrum of compound 5 


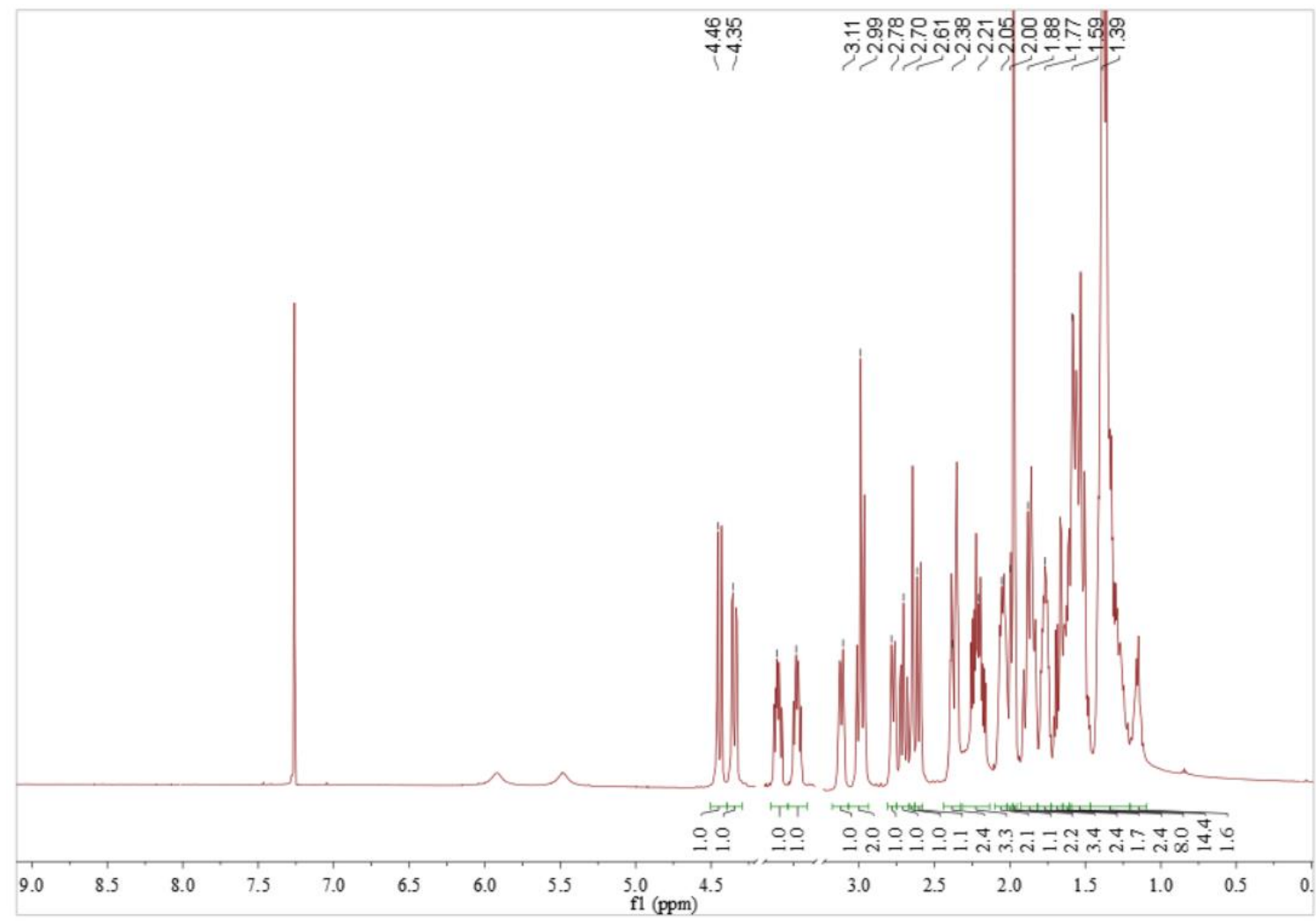

Figure S44. ${ }^{1} \mathrm{H}$ NMR spectrum of compound 5

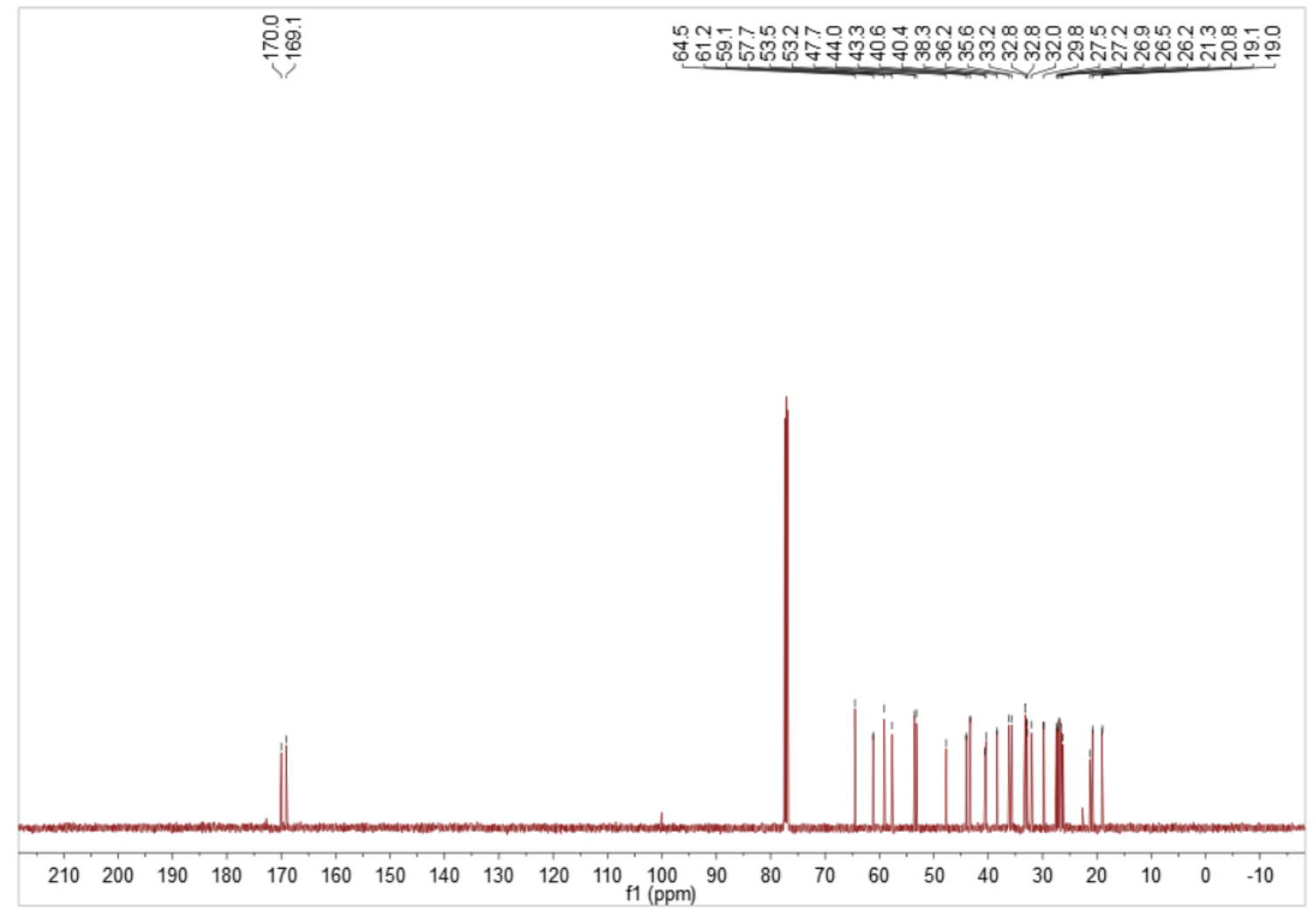

Figure S45. ${ }^{13} \mathrm{C}$ NMR spectrum of compound 5 


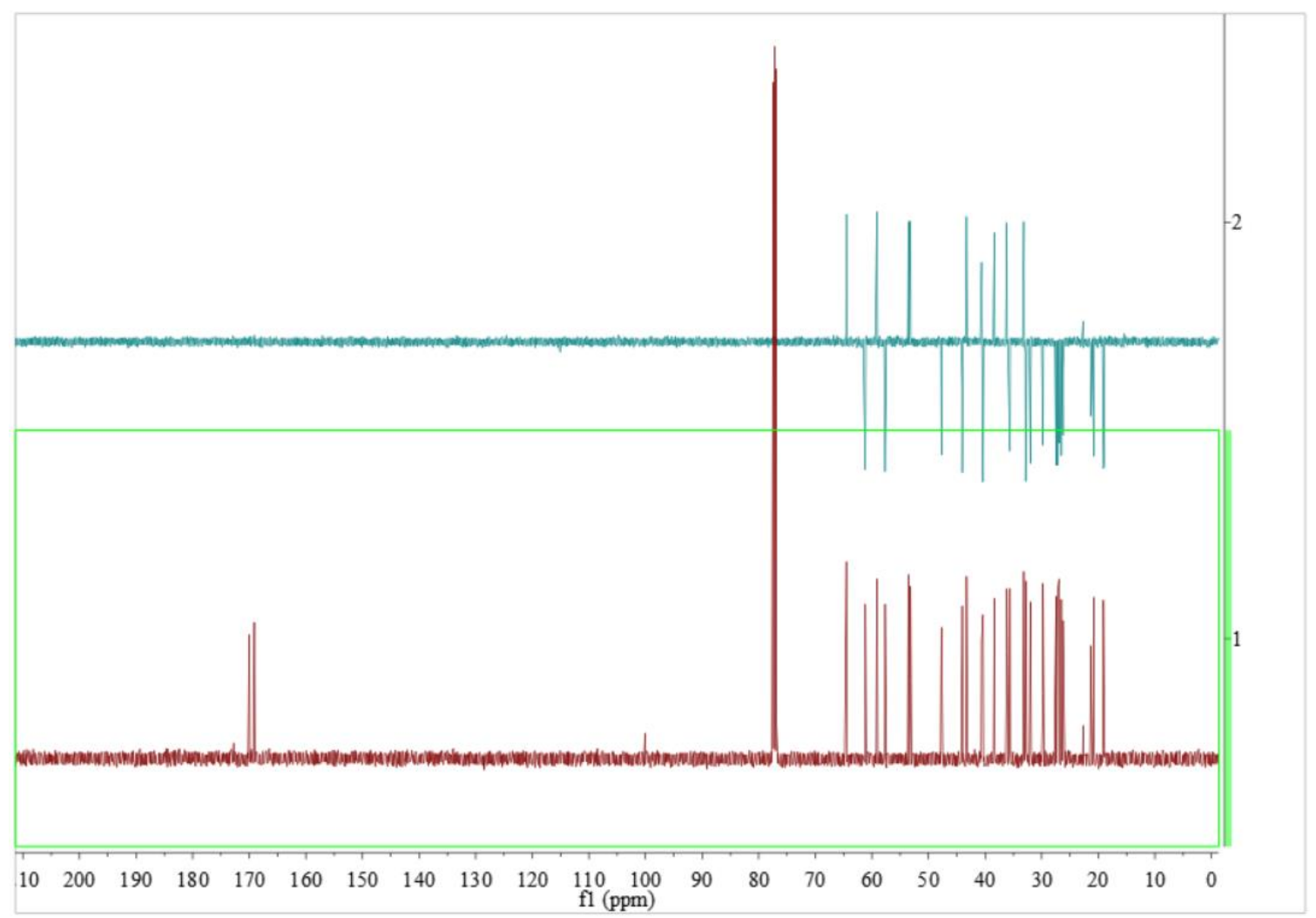

Figure S46. DEPT-135 spectrum of compound 5

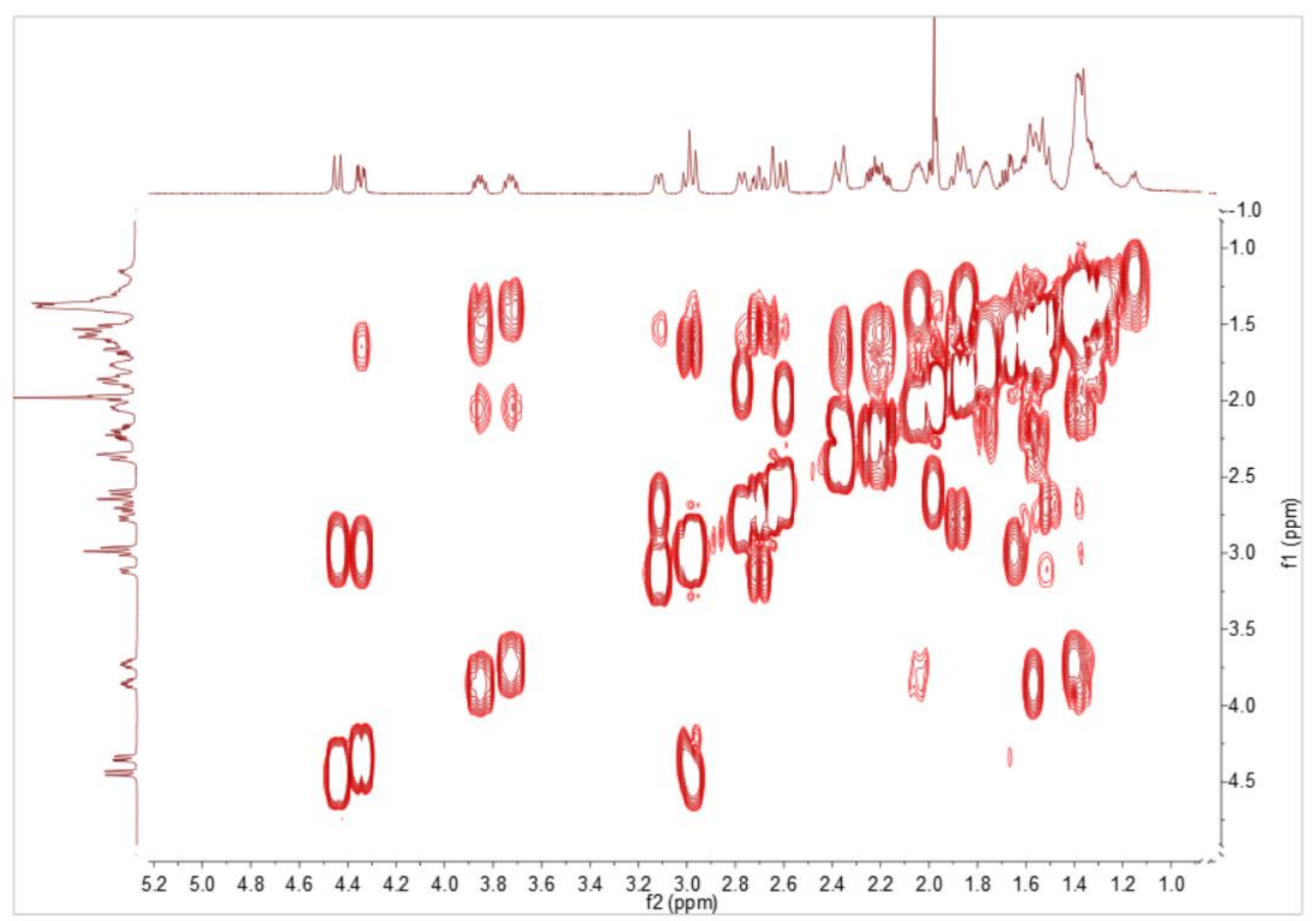

Figure S47. ${ }^{1} \mathrm{H}-{ }^{1} \mathrm{H}$ COSY spectrum of compound 5 


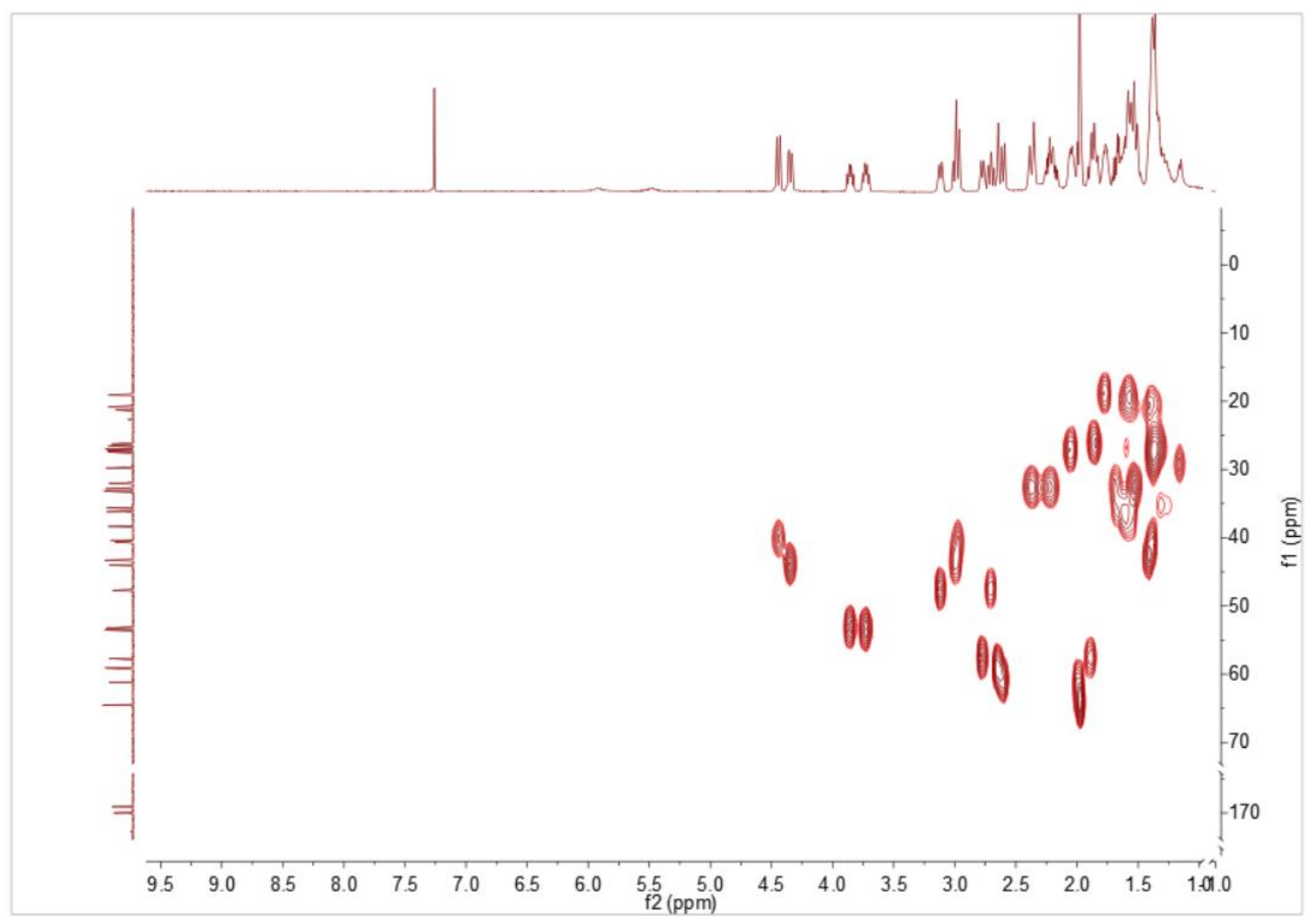

Figure S48. HSQC NMR spectrum of compound 5

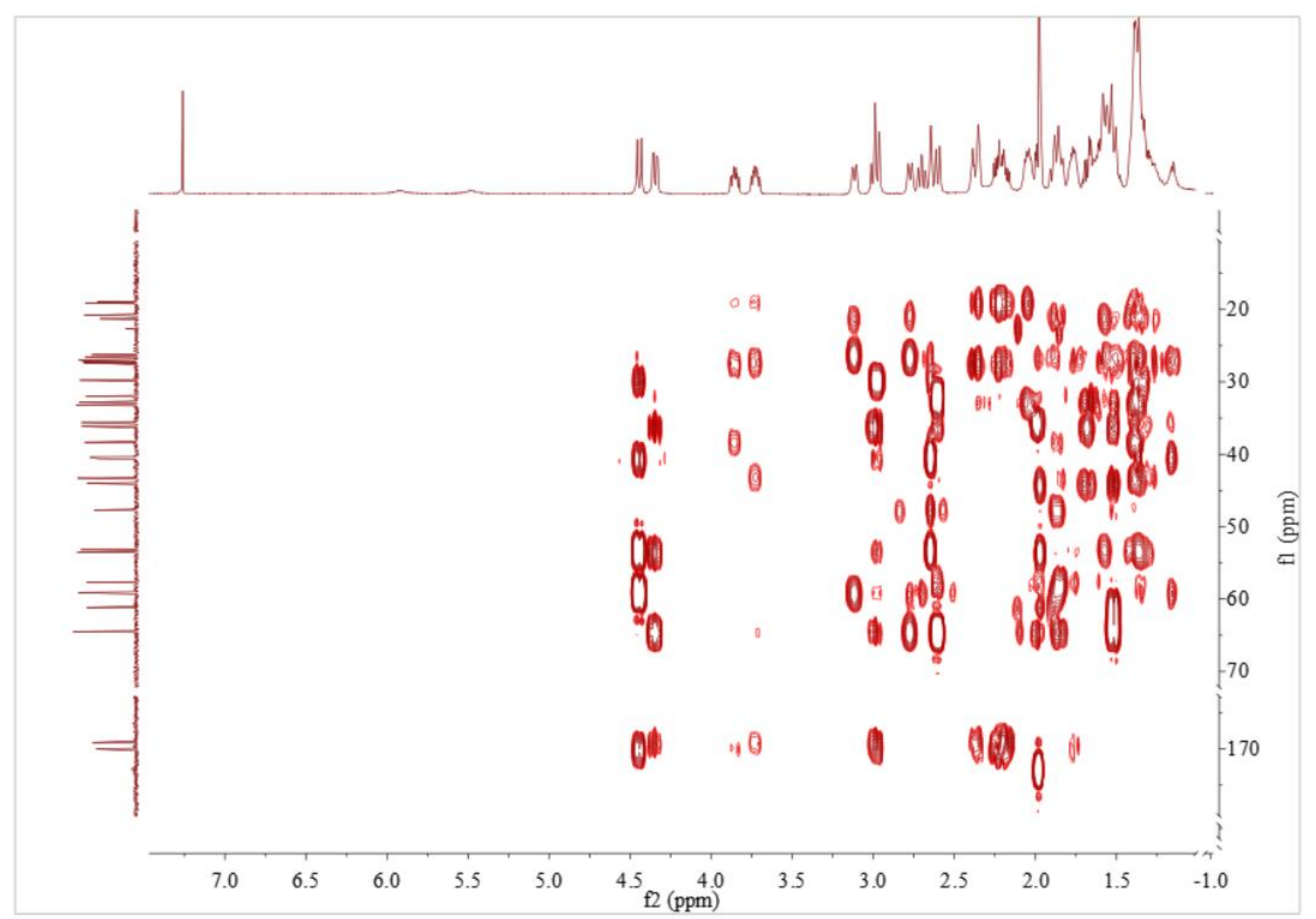

Figure S49. HMBC NMR spectrum of compound 5 


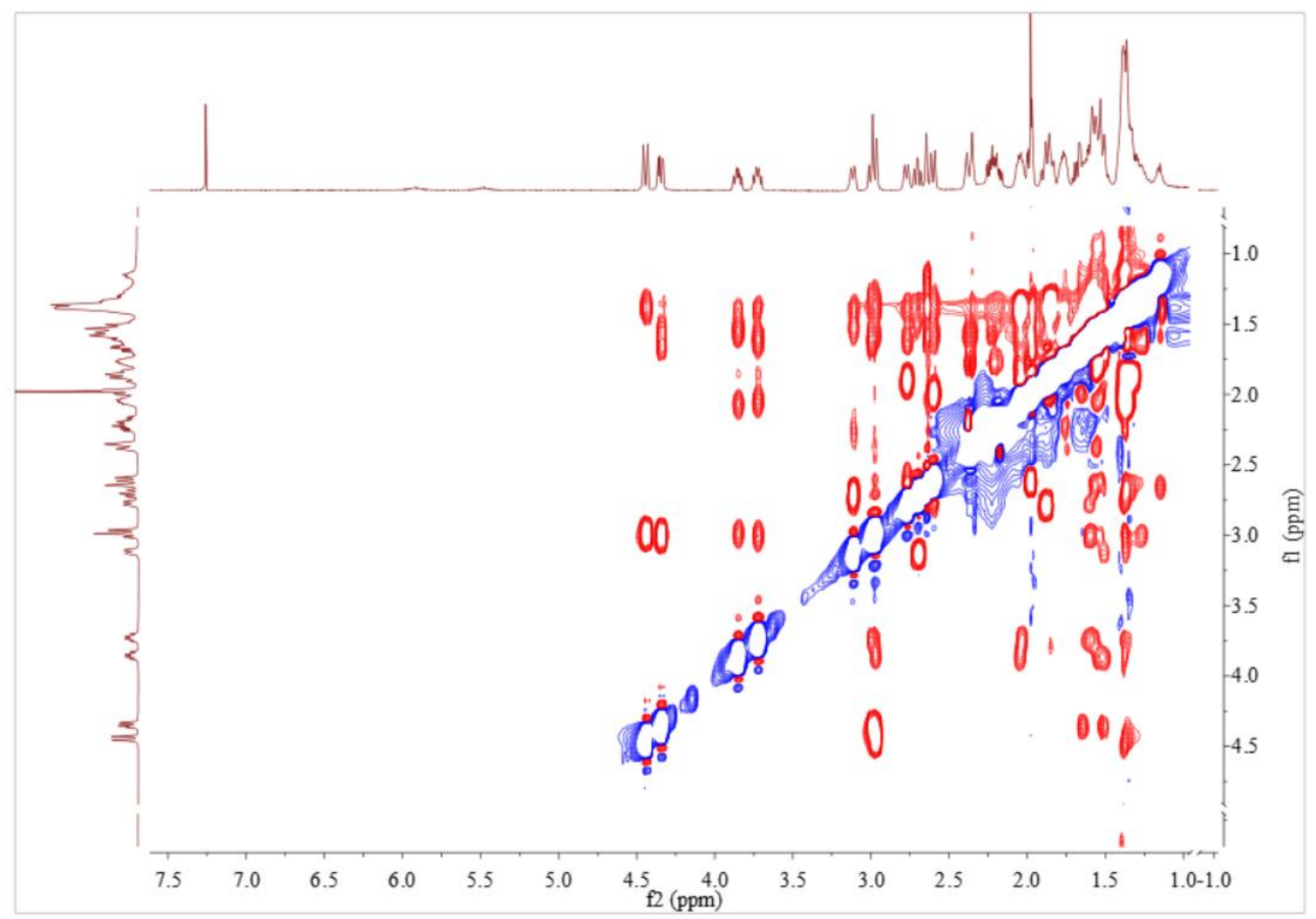

Figure S50. NOESY spectrum of compound 5 